\title{
Dienstleister der Finanzialisierung: Fragmentierte Organisation und kalkulierte Profite in der Immobilienwirtschaft
}

\author{
Sebastian Botzem • Leonhard Dobusch
}

Zusammenfassung: Die Finanzkrise von 2007 hat Fragen nach der sozialen Ordnung kapitalistischer Gesellschaften wieder in den Mittelpunkt gerückt. Vor allem in der Wirtschaftssoziologie wird ein gestiegener Einfluss von Finanzmarktakteuren und -logiken konstatiert. Die bisherige Forschung zu Finanzialisierung konzentriert sich jedoch stark auf Makrophänomene oder innerorganisationale Perspektiven. Finanzialisierungskonzepte, die ausdrücklich interorganisationale Prozesse in den Blick nehmen, sind bisher wenig entwickelt. Hier setzt der vorliegende Artikel an und untersucht exemplarisch das Geschäftsgebaren und die Finanzierungspraktiken einer europaweit tätigen Immobiliengesellschaft. Die historisch angelegte Fallstudie analysiert dabei Kooperationen zwischen unterschiedlichen Finanzdienstleistern (Banken, Makler, Notare, Wirtschaftsprüfer, Kunden) sowie die von ihnen organisierte grenzüberschreitende Verschiebung von juristischer Haftung und wirtschaftlicher Verantwortung. Der Beitrag trägt so auf zweierlei Weise zur aktuellen Finanzialisierungsdiskussion bei: Zum einen analysiert er die interorganisationale Kooperation von Dienstleistungsfirmen und erklärt ihre konstitutive Rolle in Finanzialisierungsprozessen. Zum anderen zeigt er die Bedeutung kalkulatorischer Praktiken für die Etablierung finanzialisierter Unternehmen auf.

Schlüsselwörter: Finanzialisierung · Dienstleistungssektor $\cdot$ Kalkulation · Interorganisationale Beziehungen - Immobiliensektor

\section{Financialization's services firms: fragmented organization and calculated profits accountability in the real estate sector}

\begin{abstract}
The financial crisis of 2007 has brought questions about the social order in capitalist societies back into the limelight. Economic sociology in particular emphasizes the growing influence of financial markets' actors and logics. Most research focuses on macro phenomena or intraorganizational and individual perspectives. In contrast, concepts of financialization that explicitly
\end{abstract}

(C) Springer Fachmedien Wiesbaden 2012

\section{S. Botzem $(\bowtie)$}

Wissenschaftszentrum Berlin für Sozialforschung, Reichpietschufer 50, 10785 Berlin, Deutschland

E-Mail: botzem@wzb.eu

L. Dobusch

Fachbereich Wirtschaftswissenschaft, Freie Universität Berlin, Boltzmannstr. 20, 14195 Berlin, Deutschland

E-Mail: leonhard.dobusch@fu-berlin.de 
take an inter-organizational perspective are in their infancy. Therefore, this article investigates the business and financing practices of a Europe-wide real estate firm. The historic case study shows the importance of service firms in the real estate sector and analyzes cooperation between different financial service firms (banks, estate agents, notaries, auditing firms, customers). Furthermore, it investigates how they jointly organize border-crossing and the relocation of legal liabilities and economic accountability. The article thus contributes to the current financialization debate in two ways: for one, it analyzes and explains inter-organizational cooperation between different service firms as being constitutive for financialization dynamics. For another, it points to the importance of calculative practices for establishing financialized corporations.

Keywords: Financialization $\cdot$ Services $\cdot$ Calculation $\cdot$ Interorganizational relations $\cdot$ Real estate

\section{Einleitung: Dienstleistungsunternehmen und sozioökonomischer Wandel}

Seit Ausbruch der Finanzkrise im Jahr 2007 erlangen Fragen nach der sozialen Ordnung kapitalistischer Gesellschaften wieder vermehrt an Bedeutung. Spätestens seit der Pleite von Lehman Brothers im Herbst 2008, als die Krise einen ersten Höhepunkt fand, ist der mediale Diskurs stark von den Auswirkungen der Finanzwirtschaft auf gesellschaftliche Zusammenhänge geprägt. Dabei ist das finanzielle Ausmaß der entstandenen Kosten weiterhin kaum abzusehen. Zu den betrieblichen Belastungen für Abschreibungen und Wertverlusten sowie dem folgenden Einbruch der Weltwirtschaft addieren sich finanzielle und politische Kosten rigider staatlicher Strukturanpassungsprogramme in Europa und anderswo (Streeck und Mertens 2010). Auch wenn der Ausgangspunkt der Finanzkrise in den USA verortet wird, wo das Platzen von Subprime-Krediten und der folgende Wertverlust zunächst zu Kreditausfällen privater Schuldner führte (Helleiner 2011, S. 69; auch Sorge 2011), sind die Krisenphänomene längst global spürbar.

Die Wirtschaftssoziologie richtet seit einiger Zeit gezielt das Augenmerk auf den Wandel sozialer Ordnung im Zuge von Finanzialisierungsprozessen. Es geht sowohl um veränderte Rationalitäten sozioökonomischer Zusammenhänge im Allgemeinen, vor allem eine Ausbreitung von Finanzmarktlogiken, als auch konkret um einen gestiegenen Einfluss von Finanzmarktakteuren. Veränderungsdynamiken werden oftmals bezüglich des Verhältnisses von Finanz- und Realwirtschaft diskutiert, das sich in den zurückliegenden Jahrzehnten stark gewandelt hat. Krisenhafte Erschütterungen der Finanzmärkte werden nicht mehr nur als Hinweis auf zukünftige realwirtschaftliche Veränderungen angesehen, sondern als Anhaltspunkt für einen neuen Typ finanzmarktgetriebenen Wirtschaftens gewertet:

Finanzmarkt-Krisen wurden bisher interpretiert als ein Indikator, der eine drohende Rezession in der Realökonomie antizipiert und frühzeitig signalisiert. Inzwischen sind Finanzmärkte kein Frühindikator mehr, sondern sie sind selbst zu einer Ursache für Rezessionen in der Realökonomie geworden. (Windolf 2009, S. 195; Hervorhebung im Original)

Diese Reinterpretation von Ursache-Wirkungsbeziehungen beinhaltet mehr als einen Perspektivwechsel auf sozioökonomische Zusammenhänge; sie diskutiert eine Umwälzung sozialer Ordnung, bei der nicht mehr die dingliche Produktion im Vordergrund steht, 
sondern eine „finanzielle Wertschöpfung“. Die empirische Auseinandersetzung mit der Bedeutungszunahme von Finanzmärkten, Finanzmarktakteuren und Finanzmarktlogiken hat in den letzten Jahren stark zugenommen und lässt sich je nach Forschungsperspektive unterscheiden: Finanzialisierung wird für die globale Ökonomie, einzelne Volkswirtschaften, auf Unternehmens- oder der Individualebene konstatiert (vgl. Deeg 2010; Krippner 2011; Epstein 2005; Windolf 2005a). Ausgangspunkt vieler Arbeiten zur Finanzialisierung war zunächst die Verbreitung angloamerikanischer, an kurzfristiger Profitmaximierung orientierter Praktiken, die etablierte Unternehmen aus dem Produktionssektor unter Druck setzten, ihre internen Strukturen zu ,verschlanken“ und erhöhte Profite auszuweisen. Ein gängiges Schlagwort dieser Entwicklungen ist der Aufstieg des Shareholder Value (Jürgens et al. 2000; Kädtler 2009). Die verschiedenen Ansätze eint das Bemühen, die beobachtete Zunahme von Finanzmarktlogiken, Handlungsoptionen und Akteure einzuordnen und zu erklären (vgl. Kädtler 2009). Dabei sind sowohl neue Modi der Wertschöpfung (Krippner 2005, 2011) von Bedeutung als auch der Aufstieg neuer Dienstleistungsakteure (Windolf 2008), dem wir hier besonderes Augenmerk schenken.

Der vorliegende Beitrag verdeutlicht die strukturellen Veränderungen kapitalistischen Wirtschaftens anhand einer Fallstudie der Immobilienwirtschaft. Wir rekonstruieren die Entstehung eines europäischen Immobilienkonglomerats und seine Vorbereitung auf einen Börsengang. Dabei zeigen sich die herausgehobene Bedeutung von Finanzdienstleistungsfirmen bei der Entstehung eines Unternehmens, dessen Geschäftsmodel darauf basiert, existierende Immobilien zu erwerben und diese in haftungsbeschränkten Gesellschaften zu bündeln. Mittelfristiges Ziel dieser Aktivitäten war der Börsengang des Unternehmens, der jedoch nach Ausbruch der Finanzkrise nicht mehr zustande gekommen ist.

Im Mittelpunkt unserer Analyse steht das arbeitsteilige Zusammenspiel unterschiedlicher Dienstleistungsakteure, ohne die das Konglomerat nicht existieren könnte. Unsere Studie trägt damit zu einem Perspektivwechsel der Finanzialisierungsliteratur bei und macht zwei Befunde nachvollziehbar: Erstens lässt sich zeigen, dass die Finanzialisierung im Immobiliensektor innerhalb eines Netzes eng verflochtener Organisationen erfolgt. Die arbeitsteilig erbrachten Dienste werden gezielt und bewusst in interorganisationale Beziehungen zusammengeführt. Dienstleistungsfirmen machen eine Finanzialisierung des Immobiliensektors damit erst möglich. Zweitens hebt das Fallbeispiel die Bedeutung kalkulatorischer Praktiken für die Etablierung finanzialisierter Unternehmen hervor, indem der performative Zirkel zwischen Wertgutachtern und Kreditvergabe nachvollziehbar gemacht wird. Eine weitere Konsequenz kalkulatorischer Praktiken sind die temporale und geografische Verschiebung von Haftung und Profit.

Die Rekonstruktion der Entwicklung eines europäischen Immobilienunternehmens ist auf die Beantwortung der folgenden zwei Forschungsfragen ausgelegt: Welche Rolle spielen Dienstleistungsunternehmen im Prozess der Finanzialisierung des Immobiliensektors? Auf welche Weise erbringen sie ihre Tätigkeiten, welche charakteristischen Muster der Interaktion lassen sich finden? Beide Fragen verweisen auf die Bedeutung von organisationalen Prozessen innerhalb von und zwischen Unternehmen. Damit ergänzen wir die Finanzialisierungsliteratur um eine Interorganisationsperspektive sowie um Einsichten aus der soziologischen Kalkulationsforschung. Ziel ist es, auf der Basis eines einzelnen, in seinen Strukturen aber branchentypischen Falls, einen Beitrag zum Stand der Forschung über sozio-ökonomischer Wandlungsprozesse zu leisten. 
Im konkreten Fall erklären wir die Spezifika des beobachteten Transformationsprozesses mit drei unterschiedlichen Dynamiken: Erstens, die Fragmentierung interner (Finanz-) Beziehungen und ihre Stückelung in eine Vielzahl von Dienstleistungen und Produkten, für die schon frühzeitig Gebühren in Rechnung gestellt werden; zweitens, die performative Wirkung der Kalkulation von Profiten durch die Ermittlung und Testierung von Wertgutachten durch Beratungs- und Prüfungsgesellschaften sowie deren Anerkennung durch Finanzierungsinstitutionen, allen voran Banken; und drittens, die organisationale und territoriale Verschiebung von Haftung und unternehmerischer Verantwortung zu Gunsten von Kreditgebern, Kapitalanlegern und den Dienstleistungsfirmen selbst.

Der Artikel gliedert sich in vier weitere Teile. Der folgende Abschnitt gibt einen Überblick über die Finanzialisierungsliteratur und betont die Notwendigkeit einer organisationalen Perspektive. Teil drei stellt das methodische Vorgehen dar, beschreibt das Feld, den Fall und die Datenauswertung. Anschließend wird im vierten Abschnitt der Fall IMMOFIRM rekonstruiert, indem der Organisationsaufbau dargestellt und das Geschäftsmodell sowie die organisationale Umwelt analysiert werden. Im fünften Abschnitt werden die Ergebnisse der explorativen Fallstudie zusammengefasst, eingeordnet und theoretische Implikationen diskutiert.

\section{Theoretischer Rahmen: Finanzialisierung und interorganisationale Beziehungen}

Die wachsende Bedeutung von Finanzmärkten, Finanzmarktakteuren und Finanzmarktlogiken wird vor allem in politökonomischen und wirtschaftssoziologischen Ansätzen erforscht. Sie gehen den Phänomenen der Finanzialisierung auf der Ebene der globalen Ökonomie, einzelner Volkswirtschaften und Unternehmen sowie im Alltagsleben nach. Allerdings mangelt es an konzeptioneller Klarheit, was unter Finanzialisierung zu verstehen ist.

Some writers use the term 'financialization' to mean the ascendancy of 'shareholder value' as a mode of corporate governance; some use it to refer to the growing dominance of capital market financial systems over bank-based financial systems; some follow Hilferding's lead and use the term 'financialization' to refer to the increasing political and economic power of a particular class grouping: the rentier class; for some financialization represents the explosion of financial trading with a myriad of new financial instruments. (Epstein 2005, S. 2)

Trotz der unterschiedlichen Perspektiven eint die Ansätze das Bestreben, grundlegende sozioökonomische Veränderungen zu analysieren und zu erklären. Differenzen finden sich vor allem hinsichtlich der „Lokalisierung“ des konstatierten Phänomens: Ein Teil der Literatur fokussiert auf makroinstitutionelle Entwicklungen, vor allem globale und nationale Wandlungsprozesse (Epstein 2005; Krippner 2005; Windolf 2005a; Beyer 2009; Nölke 2009; Deeg 2010; Heires und Nölke 2011; Krippner 2011), während andere Arbeiten vornehmlich die Veränderungen auf Unternehmensebene in den Blick nehmen (Münch und Guenther 2005; Froud et al. 2006; Kädtler 2009). Ebenso werden die Durchdringung der Alltagswelt und die Herausforderungen einer zunehmend finanzialisierten Ordnung für Individuen kritisch diskutiert (Erturk et al. 2007; Young 2011). 
Allgemein lässt sich festhalten, dass Finanzialisierungsansätze zunächst eine neue Perspektive auf Prozesse sozioökonomischer Transformation einnehmen. Im Fokus stehen Veränderungen der Art und Weise der Profitgenerierung. Es geht daher vor allem um neuartige, qualitativ andere Formen wirtschaftlichen Denkens und Handelns, die eine neue soziale Ordnung beschreiben und erklären. Greta Krippner bringt dies auf den Begriff des akkumulations-zentrierten ökonomischen Wandels: „I propose an accumulation-centered view of economic change, in which the focus is on where profits are generated in the economy“ (Krippner 2005, S. 177, Hervorhebung im Original). Damit sind Veränderungen im Verhältnis von Produktions- und Finanzsektor angesprochen. Finanzialisierung bezeichnet somit einen Prozess, „bei dem zunehmende Anteile der Unternehmensgewinne und Haushaltseinkünfte aus finanziellen Aktivitäten erzielt werden, im Vergleich zu jenen Anteilen, die sich direkt aus der Produktion bzw. der Lohnarbeit ergeben. Damit einher geht eine Machtverschiebung sowohl innerhalb der Unternehmen als auch zwischen ihnen, bei der der Finanzsektor dem produzierenden Sektor übergeordnet wird“ (Nölke 2009, S. 125). In Deutschland sind diese Beobachtungen eng mit dem Begriff der „,neuen Eigentümer“ verknüpft, der die Bedeutungszunahme institutioneller Investoren als Erklärung für Wandel betrachtet (Windolf 2005b). Der Aufstieg von ShareholderValue-Logiken stellt eine konkrete Form der Finanzialisierung dar, in deren Ergebnis die Handlungsspielräume von Unternehmen und ihrem Management einseitig auf die Renditeziele von Kapitalmarktakteuren als zentrale Richtgröße für strategische Entscheidungen festgelegt werden (Rappaport 1998, kritisch Jürgens et al. 2000).

Erhöhte Renditeerwartungen werden als Ursache für Veränderungen unternehmerischer Strategien interpretiert. Statt auf (traditionelles) Wachstum durch Produkt- oder Prozessinnovation zu setzen, versuchen Manager vor allem die erforderte Effizienzsteigerung durch kurzfristige - potenziell auch existenzgefährdende - Maßnahmen der Profitmaximierung zu erreichen (Windolf 2005c, S. 24). Wenngleich empirische Unternehmensstudien deutscher Großunternehmen ein Bündel interner und externer Gründe für eine zunehmende Kapitalmarktorientierung ausmachen (Höpner 2003), ist eine grundsätzlich gestiegene Bedeutung nationaler und internationaler Aktienmärkte weitgehend unstrittig. Es wird davon ausgegangen, dass Firmen sich aufgrund externer Erwartungen verändern, indem sie das Management der Unternehmung unter das Management der Finanzen subsumieren: Der Profit entspringt vermehrt einem aktiven „management of assets in order to exploit financial market opportunities“" (Boyer 2007, S. 797).

Im „Finanzmarkt-Kapitalismus“ spielen Aktienmärkte, die von institutionellen Investoren dominiert werden, die ihrerseits um Anleger buhlen und daher in einem scharfen Wettbewerb untereinander stehen, eine zentrale Rolle (Windolf 2005a). Sie sind die Orte, auf denen sich starke Konkurrenz um Anlagekapital entfaltet. Institutionelle Investoren geben diesen Druck an ihre Investitionsobjekte, zumeist börsennotierte Gesellschaften, weiter und begründen somit die Kurzfristigkeit von (Investitions-)Entscheidungen und die Maximierung von Rendite-Zielen (Windolf 2005b). Dabei ist die Konkurrenz der Investmentgesellschaften untereinander auch für diese selbst problematisch, da sie eine Spirale der Konkurrenz begründet, die die Renditeziele immer weiter nach oben schraubt. Darin ist eine Paradoxie angelegt, die für alle Finanzialisierungsansätze kennzeichnend ist: Auch wenn es individuell rational sein mag, eine möglichst hohe Rendite zu erlangen, ist der kumulierte Effekt oftmals ,,pervers“, weil systemgefährdend (Windolf 2009). 
Auch auf Ebene einzelner Unternehmen zeigt sich die Schwierigkeit, den erhöhten Renditeerwartungen langfristig zu entsprechen. Froud et al. (2006) zeigen, dass der Bedeutungszunahme von Shareholder-value-Logiken insbesondere mit Hilfe von kommunikativen Praktiken (Narrationen) entsprochen wird. Da die ständigen Erwartungen an steigende Renditen von Unternehmen operativ nicht zu erwirtschaften sind, werden die Diskrepanzen zwischen Erwartungen und unternehmerischen Ergebnissen durch Rhetorik überbrückt. Aber auch die rhetorische Bezugnahme auf erhöhte Renditen bringt Erwartungen mit sich, die eine Finanzmarktorientierung befördern.

This rhetoric adds a variety of (changing and contradictory) financial pressures on giant-firm managers, who then must accommodate them through different combinations of saying and doing with specific structural constraints (Froud et al. 2006, S. 65).

Auch hier lässt sich eine Spannung zwischen dem Handeln Einzelner und den kollektiven Effekten erkennen, denn auch der Überbietungswettbewerb der Narrationen lässt sich nicht endlos führen, auch nicht unter Rückgriff auf professionelle Agenturen und Beratungsunternehmen. Hinzu kommt, dass die Bedeutung von Finanzmärkten durch eine zwar diffuse, aber allgemein verbreitete „Finanzmarktrationalität“ gestärkt wird: Ihre Etablierung als vorrangige Begründungsordnung für wirtschaftliches Handeln ermöglicht es, die Bedürfnisse von Kapitalmarktakteuren als rational und legitim darzustellen und wird somit zu einer eigenständigen Machtressource, deren konkrete Relevanz allerdings in den jeweils spezifischen Aushandlungskonfigurationen variiert (Kädtler 2009, S. 15). Sowohl die Arbeiten von Froud et al. (2006) als auch Kädtler (2009) heben den performativen Charakter hervor, den die Bezugnahme auf Erfordernisse und Funktionslogiken von Finanzmärkten mit sich bringt (vgl. MacKenzie et al. 2007).

Wirtschaftssoziologische Performativity-Ansätze setzten sich vor allem mit den Einflüssen von finanzmathematischen Modellen und finanzwissenschaftlichen Theorien auf die wirtschaftliche Praxis auseinander (vgl. Vollmer et al. 2009). Entscheidend ist dabei nicht, dass Theoriebeiträge realwirtschaftliche Phänomene genauer vorhersagen können. Ihre Wirksamkeit liegt vor allem darin begründet, dass Marktakteure ihr Verhalten den Theorien entsprechend anpassen und sie somit „bestätigen“, wenigstens aber zu ihrer „Optimierung“ beitragen (vgl. MacKenzie und Millo 2003). Wie es gerade im Zusammenspiel fragmentierter Finanzmarktakteure zu performativen Effekten kommt, ist jedoch noch kaum erforscht.

Denn trotz vielfältiger Einblicke fehlt in der Finanzialisierungsdebatte bislang eine systematische Analyse der Gestaltung von Interorganisationsbeziehungen und der Rolle unterschiedlicher Akteursgruppen. Hier setzt der vorliegende Beitrag an, der explizit auf die konstitutive Rolle von Dienstleistungsfirmen bei der Finanzialisierung von Wohnimmobilien fokussiert. Auch wenn weitgehend darüber Einigkeit herrscht, dass Prozesse der Finanzialisierung auf unternehmerischer, sektoraler, nationaler oder globaler Ebene zusammenhängen, sind die Dynamiken und die sie tragenden Akteure bislang nur wenig erforscht. Es finden sich zwar Verweise auf die Bedeutung von wissensintensiven Industrien (Deutschmann 2005), Intermediäre des Finanzsektors (Windolf 2005c), die neue Dienstklasse des Kapitalismus (Windolf 2008) oder ,eine globale Infrastruktur von Rating-Agenturen, Beratern und Analysten“, die im Finanzmarktkapitalismus entstanden 
ist (Beyer 2009, S. 313). Eine systematische Analyse des Beitrags von Finanzdienstleistern, ihrer Kompetenzen und ihres Vorgehens steht allerdings noch aus. Hierzu möchte der vorliegende Aufsatz einen Beitrag leisten.

Unsere Studie knüpft an die oben genannten Überlegungen an und entwickelt sie mit Bezug auf interorganisationale Beziehungen weiter. Entsprechend der oben dargestellten Debatte, verstehen wir Finanzialisierung zunächst als analytischen Zugang, der eine Verortung von Profitgenerierung vornimmt. Finanzialisierung fasst jene organisationalen Veränderungen und unternehmerischen Strategien zusammen, mit denen Profitgenerierung an Finanzmarktdynamiken gekoppelt wird. In diesem Kontext gilt es zu zeigen, wie Interorganisationsbeziehungen im Detail strukturiert sind und welchen spezifischen Beitrag Dienstleistungsunternehmen dazu leisten, dass Finanzialisierung stattfindet und zunimmt. Damit wird der strategische Gehalt finanzmarktorientierter Handlungen deutlich und es kann gezeigt werden, dass Dienstleister nicht nur die Position des Finanzmanagements stärken. Vielmehr werden, wie auch im hier untersuchten Fall, Unternehmen bisweilen nur zu dem Zweck gegründet oder ausgestaltet, um die Gelegenheitsstrukturen der Finanzmärkte auszunutzen. Spezialisierte Dienstleistungsfirmen tragen dazu entscheidend bei.

Die interorganisationalen Beziehungen im Immobiliensektor sind neben den Akteuren vor allem durch Verträge und Zahlenwerke geprägt. Zahlen und Zahlungen sind nicht nur die kalkulatorische Grundlage für akkumulationsorientiertes Wirtschaften. Sie definieren zugleich die Beziehungen zwischen Organisationen. In der Soziologie werden die Eigenheiten des „organisierten Rechnens“ (Vollmer 2004) seit einiger Zeit verstärkt diskutiert, indem vor allem auf die Bedeutung kalkulatorischer Praktiken für die Aufrechterhaltung organisierter Sozialordnungen verwiesen wird. Es geht um die Wirklichkeits(re)produktion von und durch Zahlen.

Der Bedeutungszuwachs kalkulatorischer Praktiken hängt zunächst mit der Entwicklung multidivisionaler Großunternehmen und ihren spezifischen Kontroll- und Steuerungsbedürfnissen zusammen. Seit den 1970er Jahren lässt sich eine Krise dieses Unternehmenstypus konstatieren, auf die eine Desintegration großer Konglomerate folgte (Davis et al. 1994). Diese führte zu einem steigenden Bedarf an markt- und finanzorientierten Beratungsdienstleistungen, die unter anderem auch die Rationalisierung öffentlicher Dienstleistungen vorangetrieben und mit der Ermöglichung von Unternehmensübernahmen finanzmarktorientierte Strategien weiter befördert haben (Vormbusch 2004, S. 39). Die dabei entstehenden „Hyperrealitäten der Kalkulationen“(Vollmer 2003) werden maßgeblich durch Finanzdienstleister kreiert, deren Funktion es ist, (Kenn-)Zahlen zu interpretieren, zu repräsentieren und zu validieren sowie sie in Form von Graphen und Rankings darzustellen (vgl. Power 2004, S. 773).

Für den Immobiliensektor sind Intermediäre von Bedeutung, die kalkulatorische Praktiken und Strukturen bzw. „calculative infrastructures“ (Mennicken et al. 2008) beherrschen und als Dienstleistungsprodukt anbieten können. Sie bieten Verträge, Gutachten oder Testate an, die als legitim anerkannt sind. In solchen Artefakten finden sich beispielsweise die formalisierte Kalkulation von Kreditkonditionen, die Kredithöhe, Gebühren und Zinsen festlegen, oder inter- und intraorganisationale Verrechnungspreise sowie die Bestimmung von Risikoklassen und die Festsetzung von Bonuszahlungen (vgl. Mennicken und Vollmer 2007). Allerdings ist die Kalkulationsforschung, deren Wurzeln 
sowohl in den Social Studies of Finance als auch in der kritischen Accounting-Forschung liegen, ein noch recht junges Feld, in dem insbesondere noch Fragen hinsichtlich interorganisatorischer Dynamiken offen sind: „In order to gain a fuller picture about interrelations between processes of financial intermediation, organizing and market making, more investigations are called for" (Vollmer et al. 2009, S. 628).

Anhand des Immobiliensektors lassen sich erste Befunde generieren, die die organisationale Dynamik der Kalkulation bestätigen und erklären. Die Analyse hebt das Zusammenspiel von Gutachtern, die den Verkehrswert von Wohngebäuden bestimmen, und Banken, die ihre Kreditvergabe daran knüpfen, hervor. In dem Maße, in dem Banken freigiebiger mit Krediten sind, steigt die Kaufbereitschaft von Interessenten. Dies erhöht die Verkaufspreise und steigert den Verkehrswert, der als Referenz für zukünftige Wertgutachten eingesetzt wird. Es entfaltet sich ein performativer, das heißt zumindest für eine gewisse Zeit selbstbestätigender Charakter arbeitsteilig-zirkulärer Kalkulationsmodelle und -praktiken.

Eine organisationsbasierte Analyse globaler Finanzbeziehungen (vgl. Helleiner 2011, S.85) ermöglicht es, systemische Zusammenhänge vermeintlich unverbundener einzelwirtschaftlicher Entscheidungen aufzuzeigen und die kumulativen Aspekte der Finanzialisierung hervorzuheben. Ähnlich der Steuerhinterziehung gilt auch für die Finanzialisierung, dass sie häufig, in für sich jeweils rechtlich unbedeutsame Einzelakte [zerfällt], die erst in ihrer Gesamtheit zur Tatbestandsverwirklichung führen" (Leipold 2008, S. 216). Die Kombination und Kumulation von Einzelakten der Finanzialisierung begründet eine qualitativ neue Ordnung. Ortmann (2010, S.263) zufolge macht eben diese „Gesamtheit der Einzelakte... jenes neue Emergenzniveau aus, das man mit den Konzepten des korporativen Akteurs und des organisationalen Handelns soziologisch auf den Begriff bringt“".

Eine interorganisationale Perspektive legt die in der Finanzbranche übliche projektbezogene Spezialisierung offen und erlaubt zugleich die Analyse der Interaktionen unterschiedlicher Akteure. Das Geschäftsmodell der zu untersuchenden Immobiliengesellschaft verstehen wir daher als Summe integrierter Einzelteile, die wir mit Hilfe einer „Wertschöpfungs-Perspektive“ fassen. Allerdings ist die hier vorliegende Wertschöpfung eine mit Hilfe von Finanzdienstleistern herbeigeführte Kette ,finanzialisierter Wertschöpfung“. Sie unterscheidet sich sowohl von Wertschöpfungsketten der Produktion als auch von Ketten in der US-amerikanischen Eigenheimfinanzierung. Dort erfuhr die Kette der Hypothekenvergabe eine vertikale Desintegration (Jacobides 2005), die durch Spezialisierungsvorteile entstanden ist. Unterschiede in Managementstilen und organisationalen Fähigkeiten (capabilities) treiben dort die Differenzierung und Spezialisierung von Dienstleistungstätigkeiten voran und ermöglichen somit die Betrachtung der Zusammenhänge einzelner Dienstleistungsakte:

This study suggests that by shifting focus from an individual firm and from the final market for a product or service to the value chain structure and the process by which the good or service is produced, one can capture hidden but important dynamics. (Jacobides 2005, S. 490)

Eine solche Prozessperspektive steht auch in der vorliegenden Fallstudie im Mittelpunkt, wenngleich eine Wertschöpfungskette hier neu geschaffen wird, indem die Zusammen- 
führung der spezifischen Kompetenzen einzelner Dienstleister, unter Ausnutzung von Arbitragegeschäften, das Geschäftsmodell der zentralen Immobiliengesellschaft erst ermöglicht. Anders als die Finanzialisierung der Produktivwirtschaft, die zuerst im Mittelpunkt der Finanzialisierungsforschung stand, zeigt unsere Fallstudie eine zusätzliche Facette, nämlich die Schaffung von Unternehmen (hier in Vorbereitung auf einen Börsengang), deren einziges Ziel die Verzinsung von Anlagekapital ist (vgl. für eine Untersuchung ähnlich gelagerter Fälle Holm 2010). Eine dynamische Perspektive macht die (Re-)Kombination bestehender Produkte und Kompetenzen nachvollziehbar, die neue Unternehmen mit innovativen, wenngleich fragilen, Geschäftsmodellen begründen. Im Sinne von bricolage (vgl. Garud und Karnøe 2003) entstehen durch eine Rekombination vorher nicht dagewesene Produkte, Märkte und Organisationen. Für die Analyse kontinuierlicher Anpassungs-, Aushandlungs- und Gestaltungsprozesse der Finanzialisierung spielen Intermediäre als boundary spanners eine zentrale Rolle. „They aim at modulating, regulating, and sometimes controlling what kinds of resources, signals, information, and ideas pass in and pass out of the semipermeable membranes that are the boundaries of the organization" (Ansell und Weber 1999, S. 82).

Unsere Fallstudie widmet sich den Akteuren der Grenzüberwindung, charakterisiert die Art und Weise ihrer Aktivitäten und rekonstruiert ihre Bedeutung für neue Geschäftsmodelle in der Immobilienbranche. Wie oben dargelegt, verstehen wir unter Finanzialisierung einen analytischen Perspektivwechsel hinsichtlich der Verortung von Profitgenerierung, die mit einer zunehmenden Ausrichtung strategischer Unternehmensentscheidungen an den Erwartungen und Rationalitäten von Finanzmärkten einhergeht. Den Blick richten wir explizit auf die Rolle von Intermediären des Finanzsektors und ihren Dienstleistungen. Diesen Zusammenhang zeigen wir anhand konkreter Finanzierungspraktiken sowie in Bezug auf exemplarisches Geschäftsgebaren im europäischen Immobiliensektor. Dabei heben wir die Bedeutung interorganisationaler Beziehungen für die Produktentstehung, die Profitdefinition und die Rechenschaftspflichten hervor. Durch die Analyse einer fokalen Organisation (der IMMOFIRM-Gruppe) und ihrer Umweltbeziehungen wollen wir die Lücke zwischen makroinstitutionellen Finanzialisierungsansätzen auf der einen und Unternehmensstudien auf der anderen Seite zu schließen helfen.

\section{Methodik}

\subsection{Feld und Fallauswahl}

Ziel unserer kontextgebundenen Einzelfallstudie (Pettigrew 1990) ist es, spezifische Handlungs- und Organisationspraktiken mit dem Blick auf verallgemeinerbare Sektorstrukturen im europäischen Immobiliensektor anhand einer zentralen Organisation zu erklären. Als Untersuchungsobjekt fokussieren wir zu diesem Zweck auf ein Immobilienunternehmen(sgeflecht), das mit dem Ziel eines Börsenganges im Freiverkehrsmarkt (Subprime-Segment) einer europäischen Börse Mitte der 2000er Jahre gegründet worden war. Zu diesem Börsengang kam es allerdings aufgrund der Eskalation der Finanzkrise nicht mehr. Im Vordergrund unserer Untersuchung stehen demzufolge die organisationale Gestaltung, das Geschäftsgebaren und die Interorganisationsbeziehungen im Vorfeld 
des geplanten Börsenganges, der jedoch scheiterte, als die Immobilienblase, zunächst in den USA, platzte. Als Auslöser wird immer wieder die Vergabepraxis von Hypotheken an private Kreditnehmer mit geringer Bonität (,Sub-Prime Mortgages“) im Immobilienmarkt der USA genannt. Voraussetzung dafür war jedoch die anschließende Verbriefung dieser Kredite und ihre Mischung mit höherwertigen Sicherheiten, die von dritter Seite anerkannt und zertifiziert wurden, sowie die Kombination mit Absicherungsgeschäften in Form von Credit Default Swaps (CDS). Die auch für Deutschland zwischen 2004 und 2007 empirisch dokumentierte Blasenbildung in Immobilienmärkten (vgl. z. B. Claßen und Zander 2010) wird als eine der wesentlichen Ursachen für wirtschaftliche Instabilität im Sinne von Boom-and-Bust-Zyklen interpretiert (Minsky 1986/2008; Semmler und Bernard 2009) und ermöglicht so generalisierbare Aussagen über das Verhalten von Akteuren bei der Gestaltung interorganisationaler Beziehungen in einer Phase stark steigender Marktpreise für Anlagegüter.

Die im Bereich von Investment- und Zweckgesellschaften übliche verschachtelte Organisationsstruktur macht dabei die Identifikation der, im Sinne einer einzelnen und scharf abgrenzbaren, Organisation alles andere als einfach. Als Fall betrachten wir deshalb auch nicht eine einzelne (Holding-)Gesellschaft, sondern ein im Folgenden als IMMOFIRM-Gruppe bezeichnetes Firmengeflecht, bestehend aus einer Vielzahl miteinander verschachtelter Gesellschaften, die im juristischen Sinn unabhängige Organisationen darstellen. Ähnliches gilt auch für die Transaktionspartner, vor allem Dienstleistungsunternehmen, wie Banken, Prüfungsgesellschaften oder Wertgutachter, die häufig mehrfach und in verschiedenen, durch sogenannte „Chinese Walls“ getrennten, Organisationseinheiten mit IMMOFIRM in Interaktion treten. Auch wenn aus Gründen der Anonymisierung die Namen der Unternehmen nicht genannt werden können, sei an dieser Stelle darauf hingewiesen, dass es sich um etablierte Dienstleister von beträchtlicher Größe handelt, die auch nach Ausbruch der Finanzkrise noch in den jeweiligen Geschäftsbereichen (Bank, Wirtschafts- und Abrechnungsprüfung, Wertgutachten) tätig sind.

\subsection{Datenerhebung und Analyse}

Die folgende Rekonstruktion der Interorganisationsbeziehungen rund um das fokale Immobilienunternehmen basiert auf zwei Datenquellen: Erstens wurden mehrere mehrstündige Einzelinterviews mit zwei in leitender Funktion tätigen Mitarbeitern von IMMOFIRM geführt, aufgezeichnet und transkribiert. Mit einem der beiden Interviewpartner fanden insgesamt drei Interviews statt, wobei das dritte Interview an Hand einer vorläufigen Fassung dieses Papiers erfolgte und vor allem der Korrektur und Ergänzung diente. Die Angaben dieser beiden Hauptinformanten wurden in der Folge in weiteren informellen Gesprächen mit im Unternehmen zur fraglichen Zeit tätigen Personen hinsichtlich ihrer Plausibilität überprüft. Um persönliche Konsequenzen für die Interviewpartner zu vermeiden, wurden Unternehmen und Akteure bestmöglich anonymisiert und sämtliche veröffentlichten Zitate vor Veröffentlichung den betroffenen Personen vorgelegt. Zweitens, vor allem als Hintergrundinformation, stützt sich unsere Darstellung auf die Presseberichterstattung zu dem untersuchten Fall.

In der Datenanalyse verfolgten wir ein dreistufiges Vorgehen: In einem ersten Schritt versuchten wir, auf Basis der vorhandenen Informationen die organisationale Struktur 
von IMMOFIRM zu rekonstruieren, gefolgt von einer schematischen Darstellung der relevanten Interaktionspartner und -beziehungen. Im dritten Analyseschritt wurde, darauf aufbauend, der Prozess der gewöhnlichen Geschäftstätigkeit analysiert. Zum besseren Verständnis wurde diese Reihenfolge in der nun folgenden Darstellung der Ergebnisse adaptiert; das Netzwerk aus Interaktionsbeziehungen wird erst nach Erläuterung der Organisationsstrukturen und Geschäftsprozesse dargestellt.

\section{Der Fall (von) IMMOFIRM}

Im Vordergrund der folgenden Darstellung steht eine möglichst genaue Charakterisierung der (inter-)organisationalen Strukturen und Geschäftsprozesse des untersuchten Falls. Sie beschreibt ein europaweit tätiges Immobilienunternehmensgeflecht rund um das Unternehmen IMMOFIRM. Kern des Geschäftsmodells war der Aufkauf bestehender, vor allem in Deutschland befindlicher großer Wohnimmobilien, die mit fremdfinanziertem Kapital erworben und in Einzelfirmen erfasst wurden. Die einzelnen Objekte (in jeweils rechtlich eigenständigen Objektgesellschaften) wurden in einer Dachgesellschaft gebündelt, um sie zu einem späteren Zeitpunkt als gemeinsames Immobilienunternehmen an eine europäische Börse zu bringen. Der erhoffte Börsengang (Initial Public Offering), der aufgrund der Finanzkrise von 2007 nicht mehr stattfand, sollte die Unternehmenstätigkeiten refinanzieren und, zusätzlich zu den während der Vorbereitung auf den Börsengang an die Dienstleistungsunternehmen gezahlten Gebühren und Honorare, weiteren Profit generieren. Das meiste Kapital wurde von einer großen europäischen Bank (wenn auch von unterschiedlichen Abteilungen und unter unterschiedlichen Titeln) bereitgestellt, ein geringerer Teil wurde von mehreren weiteren Banken sowie von Privatinvestoren beigetragen.

\subsection{Die Struktur der IMMOFIRM}

Der von der IMMOFIRM-Gruppe verwaltete Immobilienbestand mit einem Bilanzwert im Milliarden-Euro-Bereich verteilte sich auf insgesamt 80 Objektgesellschaften (GmbH \& Co KGs, s. Anhang A), die wiederum in Holding-Gesellschaften (GmbHs) zusammengefasst oder mit diesen verschränkt waren. Es wurde mit Hilfe großer Rechtsanwaltskanzleien eine bewusst komplexe Struktur geschaffen, deren Ziel es war, Steuern und Haftungsrisiken zu minimieren sowie über sogenannte „Share Deals“ (Verkauf nicht der Immobilie, sondern der Gesellschaften) Investmentpakete zum Weiterverkauf zu schüren. ${ }^{1}$

Konstruktion war eine $\mathrm{GmbH} \& \mathrm{Co} \mathrm{KG}$, die eigentlich Eigentümerin war des jeweiligen Objekts. Komplementärin der $\mathrm{GmbH} \& \mathrm{Co} \mathrm{KG}$ war jeweils eine gleichnamige $\mathrm{GmbH}$. Diese GmbH war Tochter einer Holding und diese Holding war gleichzeitig Kommanditistin in dieser $\mathrm{GmbH} \& \mathrm{Co} \mathrm{KG}$. Also ging irgendwie alles sehr stark im Kreis. (Interview)

1 Bei diesen „Share Deals“ fällt unter bestimmten Voraussetzungen (z. B. mindestens zwei Investoren) keine Grunderwerbsteuer an. 
Während die Objektgesellschaften ihren Sitz am Ort der Objekte, also in der großen Mehrzahl in Deutschland hatten, standen ihre jeweiligen Muttergesellschaften, die IMMOFIRM-Holdings, ihrerseits wiederum im Eigentum einer Offshore-Gesellschaft (Oasis Ltd.) mit Sitz in der innereuropäischen Steueroase OASIS.

Vor allem die Objektgesellschaften, aber auch die Offshore-Gesellschaft IMMOFIRM Central Holding Ltd. entrichteten regelmäßige und auf die Investmenthöhe bezogene Gebühren, sogenannte „Management \& Structuring Fees“, an eine weitere Offshore-Gesellschaft, die für das Beteiligungsmanagement zuständig war und der Gewinnausschüttung an die Eigentümer diente (Investment Management Holding Ltd.).

Abbildung 1 liefert eine vereinfachte Darstellung des Firmengeflechts der IMMOFIRM-Gruppe. Die abgebildete Vielfalt an Objektgesellschaften diente dazu, möglichst flexibel einzelne Teile des Immobilienportfolios weiterveräußern oder untergehen lassen zu können. Mehrere Holding-GmbHs als Eigentümer des GmbH-Komplementärs in der $\mathrm{GmbH} \& \mathrm{Co} \mathrm{KG}$ waren wiederum notwendig, um die Zinsschrankenregelung zu umgehen, die ein Limit für die Höhe von steuermindernden Zinsaufwänden festlegt (vgl. $\S \S 4 \mathrm{~h}$ EStG iVm $\S 8$ KStG). Dreh- und Angelpunkt der gesamten Konstruktion war die Investment Management Holding Ltd. mit Sitz in einer europäischen Steueroase, die mehrheitlich im Eigentum jener Investoren stand, die für Gründung, Aufbau und Strategie der Gruppe verantwortlich zeichneten. Dort lieferten die einzelnen Gesellschaften Gebühren ab, wurden Gewinne ausgeschüttet und Aufträge für das operative Management vergeben.

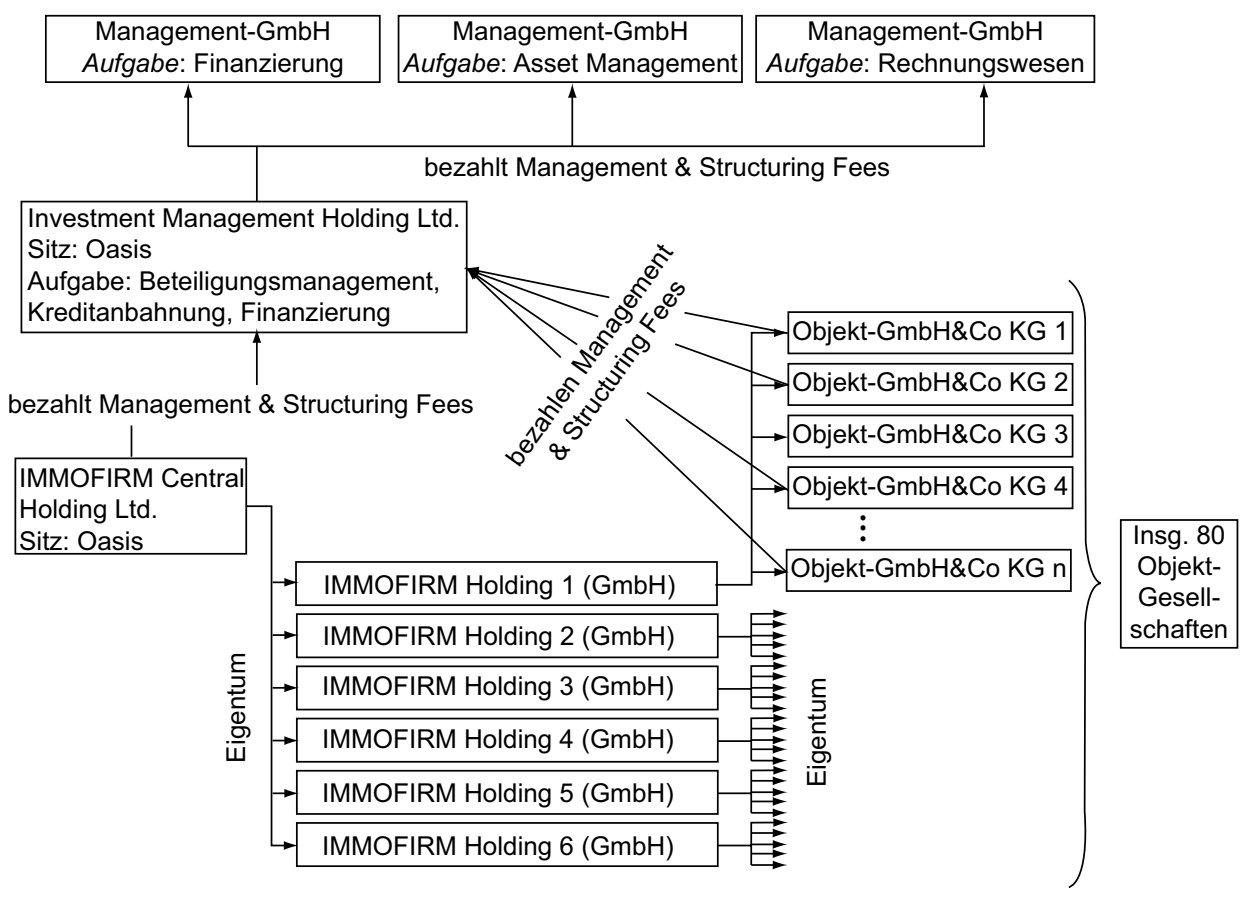

Abb. 1: Organisationale Struktur der IMMOFIRM-Gruppe. (Quelle: Eigene Darstellung) 
Die Höhe dieser Gebühren von 2,8\% der Investitionssumme war beträchtlich, aber noch im Bereich des Branchenüblichen (,die Art und Weise der Konstruktion ist völlig normal“", Interview) und mit Einverständnis der finanzierenden Bank(en) vereinbart. Hervorzuheben ist hier, dass von den einzelnen Immobilienobjekten schon ab Zeitpunkt des Erwerbs Finanzmittel, deklariert als Gebühren, in den Zentralbereich geleitet wurden. Dieser Gewinn fiel in einer speziellen Holding in der Steueroase an.

Das Tagesgeschäft in Form von Finanzierungsanbahnung, Asset Management und Rechnungswesen wurde allerdings nicht von dieser Beteiligungsgesellschaft, sondern von drei nach den Aufgabenbereichen Finanzierung, Asset Management (also insbesondere Verwaltung des Immobilienbestands) und Rechnungswesen gegliederten Gesellschaften mit Sitz in drei verschiedenen Staaten der EU abgewickelt. Die dafür entrichteten Gebühren waren gerade hoch genug, um den gewinnorientierten Charakter dieser drei Management-GmbHs zu belegen.

Die Komplexität der intraorganisationalen Struktur der IMMOFIRM-Gruppe diente im Wesentlichen der Steuervermeidung und der Haftungsbeschränkung. Letztere geht mit dem Umstand einher, dass

diese Komplementärin als einzige persönlich haftende Gesellschaft drin ist, die 25000 Euro Eigenkapital hat und das war es. Mehr kriegt die auch nicht. Und so kann jedes einzelne von diesen Dingern untergehen, ohne dass es irgendetwas anderes mitreißt. (Interview)

Steuerrechtlich führte die umsatzbezogene Verrechnung von Gebühren durch OffshoreGesellschaften zu dem gewünschten Effekt, dass steuermindernde Aufwände onshore, im konkreten Fall also in Deutschland, und Erträge größtenteils nur minimal versteuert offshore, im konkreten Fall in der Beteiligungsgesellschaft Investment Management Holding Ltd., anfielen. ${ }^{2}$ Weiteres Indiz für eine bloß formalrechtliche Trennung der verschiedenen Gesellschaften aus steuer- und haftungsrechtlichen Gründen ist, dass diese Trennung zur Liquiditätsoptimierung im Tagesgeschäft de facto ignoriert wurde, weil „man im Prinzip ein Cash-Pooling lebte, halt mit zahlreichen gegenseitigen Kreditverträgen" (Interview).

\subsection{Der IMMOFIRM Geschäftsprozess}

Ziel der gesamten Firmenkonstruktion war der Aufbau eines Immobilienportfolios mit ausreichender Größe, um einen Börsengang im Subprime-Segment einer europäischen Börse zu ermöglichen, unter Einsatz eines Minimums an Eigenkapital. Wobei Eigenkapital - „equity“ - hier relativ zu verstehen ist, denn „Eigenkapital ist, in der Branche, alles, was nicht von einem selbst kommt" (Interview). Im konkreten Fall führte das beispielsweise dazu, dass bisweilen 100\% eines Objekts mit Mitteln eines einzigen Geldgebers fremdfinanziert wurden, weil in der finanzierenden Bank unterschiedliche Abteilungen

2 Tatsächlich erfordert die steuerbefreiende Wirkung eine noch etwas kompliziertere Konstruktion unter Rückgriff auf Treuhänder, denn ,es zahlen nur solche Firmen keine Steuer, die von einem Nicht-OASIS-Resident geeignet werden. Aber wenn ein Treuhänder das verwaltet, dann ist das steuerfrei“ (Interview). 


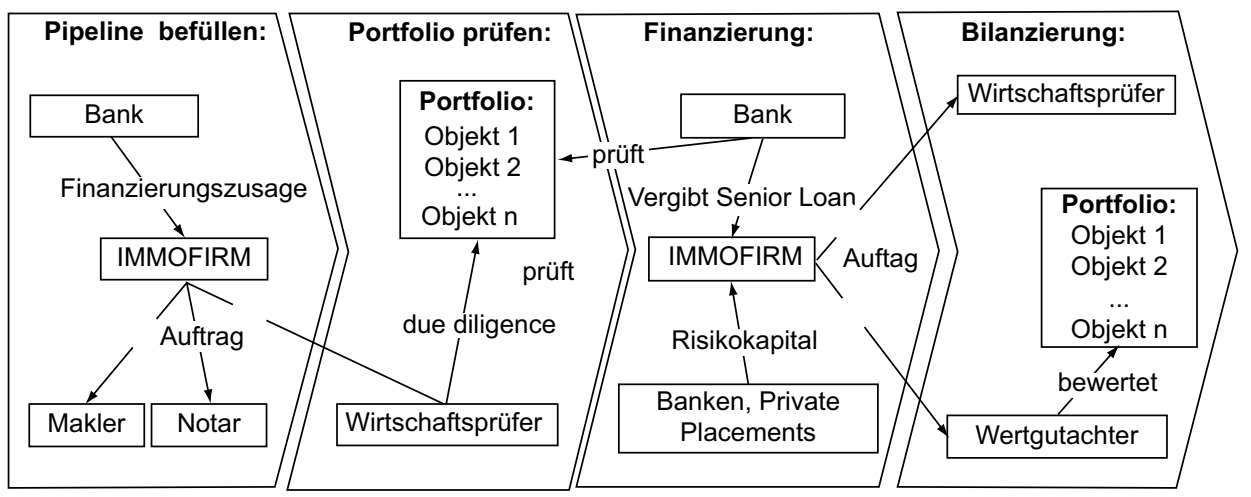

Abb. 2: Geschäftsprozessbetrachtung des IMMOFIRM-Falles. (Quelle: Eigene Darstellung)

zuständig waren und so die Mittel der Investment-Abteilung von der Hypothekarkreditabteilung als ,equity“ kalkuliert wurden.

Die gewöhnliche Geschäftstätigkeit der IMMOFIRM bestand aus Zyklen von Finanzierungsrunden (benannt nach Märchenfiguren oder Urlaubsorten, z.B. Troll oder Mallorca) mit dem Ziel, den Immobilienbestand zu erhöhen. Abbildung 2 liefert eine schematische Darstellung des Ablaufs einer solchen Finanzierungsrunde. In einem ersten Schritt („Pipeline befüllen“ in Abb. 2) wurden vom Hauptbankpartner prinzipielle, also noch unverbindliche Finanzierungszusagen für den Ankauf von Immobilien einer gewissen Kategorie (z. B. Wohnimmobilien im Niedrigmietsegment) und in einem bestimmten Ausmaß (meist zwischen 150 und 200 Mio. $€$ ) eingeholt.

Diese schriftliche Finanzierungszusage erlaubt, Makler und Notare damit zu beauftragen, entsprechende, am Markt verfügbare Immobilien anzukaufen, bis ungefähr das mit der Bank vereinbarte Volumen beisammen war, wobei sämtliche notarisierten Kaufverträge unter der auflösenden Bedingung der Finanzierung stehen. Dieser, als Befüllen der „Pipeline“ bezeichnete Prozess, wird in der Regel unter großem Zeitdruck abgewickelt:

Aber im Prinzip, so wie es damals war, als alles sehr schnell gehen musste, wurde auch immer sehr viel Zeitdruck darauf gelegt, je schneller man das ganze kauft, finanziert und fertig hat, desto besser. (Interview)

Hintergrund für den Zeitdruck war die durchaus reflektierte Beobachtung oder Erkenntnis, dass es sich bei der damaligen Marktsituation um eine Immobilienblase gehandelt hat. Erkennbar wird dies an Aussagen wie „Der Markt ist bullish!“ oder „The window of opportunity is closing!“”, die konkrete Folgen für die Einkaufspolitik hatten:

Die paradoxe Situation ist, dass man quasi gefeiert hat, dass man so gut war, dass man soviel kaufen konnte. Etwas Einkaufen ist ja jetzt nicht eine besondere Leistung. Wenn Du mir das Geld gibst, kaufe ich ein Geschäft leer. Aber, im Prinzip, was Performance ausgemacht hat, war, so schnell wie möglich soviel wie möglich zu kaufen. Das Geld war eine Zeit lang... nicht wirklich ein Problem. (Interview)

Das auf diese Weise erstellte Portfolio wurde im nächsten Schritt („Portfolio prüfen“ in Abb.2) von einem Wirtschaftsprüfer (,War meiner Meinung nach eine sehr oberfläch- 
liche Arbeit“, Interview) und einem technischen Sachverständigen einer Due-diligencePrüfung unterzogen. Auf Basis dieser Prüfung erfolgte dann („Finanzierung“ in Abb.2) die Vergabe des „Senior Loan“, ein klassischer Kredit, grundschuldbesichert im ersten Rang, in einem Ausmaß von ca. $80 \%$ der Gesamtkosten der Transaktion, also inklusive Makler- und Notariatsgebühren sowie Vertragserrichtungsgebühren und Kosten für due diligence. Die Laufzeit dieser Senior Loans beträgt rund 5-10 Jahre und ist in den meisten Fällen endfällig ohne nennenswerte Tilgungsleistung (,Die Fiktion im Markt ist immer die, das refinanzierst du dann sowieso“, Interview). Die Zinsen für diesen Senior Loan werden in der Folge nach den Betriebskosten prioritär aus den laufenden Mieteinnahmen der jeweiligen Objekte finanziert. Auf Seiten der Bank, des Senior Lenders, wurden diese Kredite aber ,natürlich auch verbrieft und weitergereicht“ (Interview).

Entscheidend zur weiteren Reduktion des notwendigen Eigenkapitals (aus Perspektive der IMMOFIRM) ist in der Folge die Gewinnung von Investoren für die restlichen ca. $20 \%$ des nicht vom Senior Loan gedeckten Finanzierungsbedarfs. Im Fall der IMMOFIRM wurde dieses Kapital auf verschiedene Arten aufgebracht. In wirtschaftlicher Hinsicht wurde das rechtliche Fremdkapital in fast allen Fällen (im Sinne der Risikoexposition und der erfolgsorientierten Konstruktion der Verzinsung) als Eigenkapital kalkuliert:

- Mezzaninekredit ${ }^{3}$ von einer anderen, auf risikoreiche Investitionen spezialisierten Abteilung jener Bank, die in vielen Fällen auch als Senior Lender fungierte.

- kleinere Kredite anderer Banken.

- Private Placements privater Investoren und Anleihen.

- Refinanzierung ,alter“ Senior Loans mit höherem Kreditvolumen auf Grund von gutachterlicher Neubewertung des Immobilienbestands.

Insbesondere die letztgenannte Maßnahme, die teilweise Deckung von Finanzierungslücken aus Mitteln späterer Senior Loans, ähnelt bereits einer pyramidenspielartigen, von Minsky (1986/2008) als „Ponzi finance“ bezeichneten Konstruktion.

Das Zauberwort war Refinanzierung. Diese $80 \%, \ldots$ die eine Bank bereit war zu finanzieren, diese 80 oder $85 \%$ waren ,Loan to Value'. Das heißt, wenn ich eine Immobilie habe, die ich einmal um 100 eingekauft habe und ein Marktgutachten ergibt, und die Bank glaubt das oder akzeptiert das, dass es jetzt 150 Wert ist, dann sind sie plötzlich bereit, dass sie 120 [Prozent des Ursprungsbetrags] darauf zahlen, als Kredit. (Interview)

Die auf diese Weise zu einem späteren Zeitpunkt zusätzlich erhaltenen Mittel wurden, abzüglich anfallender Gebühren, verwendet, um „die Equity-Löcher der nächsten Projekte [zu] finanzieren“ (Interview). Im Ergebnis hat diese Finanzierungsstrategie dazu geführt, dass es

3 Als Mezzaninekredite werden solche tendenziell risikobehafteten Kredite bezeichnet, die eine Art Mittelding zwischen Eigen- und Fremdkapital darstellen. Sie dienen häufig der Überbrückung der Phase zwischen Erstfinanzierung und Refinanzierung und sind in der Regel hochverzinslich (Euribor plus 5 bis über 10\% im Vergleich zu plus 1,5\% für den Senior Loan) und enthalten Klauseln, die zusätzliche Zahlungen im Falle eines Verkaufs oder Börsengangs vorsehen (sogenannte „Equity Kicker“). 
Eigenkapital im eigentlichen Sinn, eingezahltes Eigenkapital,... in der ganzen Firma keinen Cent mehr gab, als das Gesetz vorgeschrieben hat. Niemals. Also jede einzelne beteiligte $\mathrm{GmbH}$ hat 25000 Euro. Ende. (Interview)

Am Ende des Prozesses stehen Bewertung und Bilanzierung des zusammengestellten Portfolios. Die durch zusätzlich beigebrachte Wertgutachten dokumentierten Wertanstiege von Objekten werden in der Bilanzierung berücksichtigt. Das Gegenstück zum „loan to value“ durch die Bank im Bereich der Refinanzierung, ist „fair value“ im Bereich der Bilanzierung („Bilanzierung“ in Abb.2). Der geprüfte Abschluss nach den Kriterien der International Financial Reporting Standards (IFRS) war dabei für diese Refinanzierungsprozesse von keiner Bedeutung („Die Banken haben sich für das in der IFRS-Bilanz ausgewiesene Eigenkapital nicht interessiert, sondern machten sich ihr eigenes Bild“, Interview). Allerdings waren Kalkulationen auf Basis internationaler Rechnungslegungsstandards für den geplanten Börsengang erforderlich, denn die EU schreibt diese Standards für Konzernabschlüsse seit 2005 europaweit vor:

Man brauchte einen IFRS-Abschluss, damit man an die Börse gehen kann mit dem Teilchen. (Interview)

Internationale Rechnungslegungsstandards sind ein wichtiges Signal an international tätige Investoren und erfüllen daher eine entscheidende Legitimationsfunktion. Zudem erlaubt die Marktwertorientierung der Standards (fair value accounting) den Ausweis eines maximalen Unternehmenswerts in Zeiten einer Immobilienblase, so jedenfalls die Annahme vor der Finanzkrise (vgl. Nölke und Perry 2008; Botzem 2012) und auch der Fall bei IMMOFIRM.

\subsection{Das interorganisationale Netzwerk rund um IMMOFIRM}

Voraussetzung für das dargelegte Geschäftsmodell war das internationale und interorganisationale Zusammenwirken einer Vielfalt von Akteuren, das im Folgenden näher beleuchtet wird. Die Kalkulation von Profit war in ein Netz verschiedener externer Dienstleistungsunternehmen eingebettet oder wurde von diesen erbracht. Besonders bedeutsam sind Bewertungs- und Prüfungsgesellschaften, die die in den Bilanzen ausgewiesenen Werte ansetzen und testieren. Auch wenn diese Tätigkeiten innerhalb eines weltweit tätigen Netzwerks von nur einer großen Prüfungsgesellschaft durchgeführt wurden, waren die einzelnen Aufgaben auf unterschiedliche Büros in verschiedenen Ländern verteilt. Die extern eingeholten Wertgutachten wurden von einem weiteren Dienstleistungsunternehmen (Wertgutachter) mit Sitz in London erstellt. Auch das laufende Geschäftsgebaren (Mietabrechnungen und Zinsfluss) wurde nicht von den finanzierenden Banken selbst geprüft, sondern von weiteren Dienstleistern (sogenanntes „Loan Servicing“). Abbildung 3 fasst die zentralen Dienstleister und deren Zusammenwirken rund um die IMMOFIRM-Gruppe zusammen.

Für alle diese Tätigkeiten wurden im Verlauf der Vorbereitungen des Börsengangs Gebühren entrichtet. Durch relativ hohe Krediterrichtungsgebühren unter verschiedenen Titeln (z. B. für die Strukturierung, Errichtung, Disagios, d.h. Abschlag vom Nennwert des Kredits) wurden, insbesondere im Mezzaninebereich, große Bestandteile der Verzinsung an den Beginn der Kreditlaufzeit verschoben. Dies ist wiederum relevant für die 
Bonuszahlung der zuständigen Bankmanager, weil diese hohen, anfänglichen Zinszahlungen als Gewinn ausgewiesen werden. ${ }^{4}$

Entscheidend ist, dass die Werte zwar zwischen den beteiligten Unternehmen und Personen als plausibel anerkannt wurden, ihre Annahme aber auf Einschätzungen, im Sinne allgemein anerkannter Interpretationen, der Marktlage beruhten. Im Immobilienbereich kommt hierbei allerdings hinzu, dass die Marktlage nur aus Vergleichstransaktionen abgeleitet werden kann und auf Grund der Idiosynkrasien der gehandelten Immobilien nicht auf exakten Entsprechungen basieren. Eine tatsächliche Vergewisserung über die errechneten Beträge war dagegen kaum möglich - und wohl auch nicht erwünscht. Dies hängt nicht zuletzt damit zusammen, dass sich die Gebühren für die Geschäfte zu einem wesentlichen Teil aus dem „Wert“ des Portfolios ergaben. Daher profitieren alle beteiligten Gesellschaften und Personen von der Ansetzung möglichst hoher Werte.

Für den Fall der IMMOFIRM-Gruppe lässt sich dieser paradoxe Umstand, dass hohe Bewertungen in der Regel nicht nur im Interesse des Gutachters, sondern auch des Käufers (!) sind, wie folgt illustrieren:

Beim Einkaufen hat der Auftraggeber, das ist der Eigentümer oder der zukünftige Eigentümer, immer ein Interesse, das möglichst hoch zu haben, weil Du kriegst ja Loan to Value. Also entweder [der Gutachter] rechtfertigt einen Preis, den Du schon verhandelt hast. Wenn er unter dem Preis ist, im Wert, den Du verhandelt hast, musst Du entweder nachverhandeln oder das Objekt fallen lassen, weil die Bank sagt, ,Sie zahlen zuviel!' Wenn er aber mehr begutachtet, als Du an Preis vereinbart hast, ist das für Dich natürlich gut, weil Du kriegst ja Loan to Value, und hast was über. (Interview)

Auffällig ist außerdem, dass einzelne Unternehmen mit Hilfe einer sogenannten „Chinese Wall" in fiktive Einheiten untergliedert werden (vgl. Abb. 3). Im Idealfall handelt es sich also um unterschiedliche und unabhängige Abteilungen eines Unternehmens, zwischen denen es keinen Informationsaustausch geben soll, um Interessenskonflikte zu vermeiden. Ob oder inwieweit dies tatsächlich der Fall ist, wäre durch weitere empirische Forschung zu erheben.

Im Fall der IMMOFIRM-Gruppe finden sich diesbezüglich bestenfalls Indizien, wie sich an Hand von Konflikten um die Bewertung im Zuge der Erstellung der Bilanz für 2006 zeigen lässt:

[Der Wirtschaftsprüfer] hat auch irgendwo in München einen Immobiliengutachter sitzen, halt ein sehr konservativer Deutscher, der sagt, also irgendwie ist das alles korrekt gerechnet, ja. Aber unter dem Strich zahlt das kein Mensch. Die [Wertgutachter Firma ABCD] sagt, das ist alles richtig gerechnet und ob das wer zahlt oder nicht, das ist eine Beweisfrage.... Und das witzige war, aufgelöst hat sich das dadurch, dass die Deutschen gesagt haben, naja, an und für sich ist ja [Wirtschaftsprüfer in] OASIS beauftragt, weil es ist ja eine OASIS-Gesellschaft. Die müssen ja

4 Diese Gebühren werden also in den vergebenen Kredit eingerechnet, aber auf Seite der Bank als Umsatzerlös verbucht. Damit dies darstellbar bleibt, ist aber wiederum eine besonders gute Bewertung des zugrundeliegenden Assets erforderlich. 


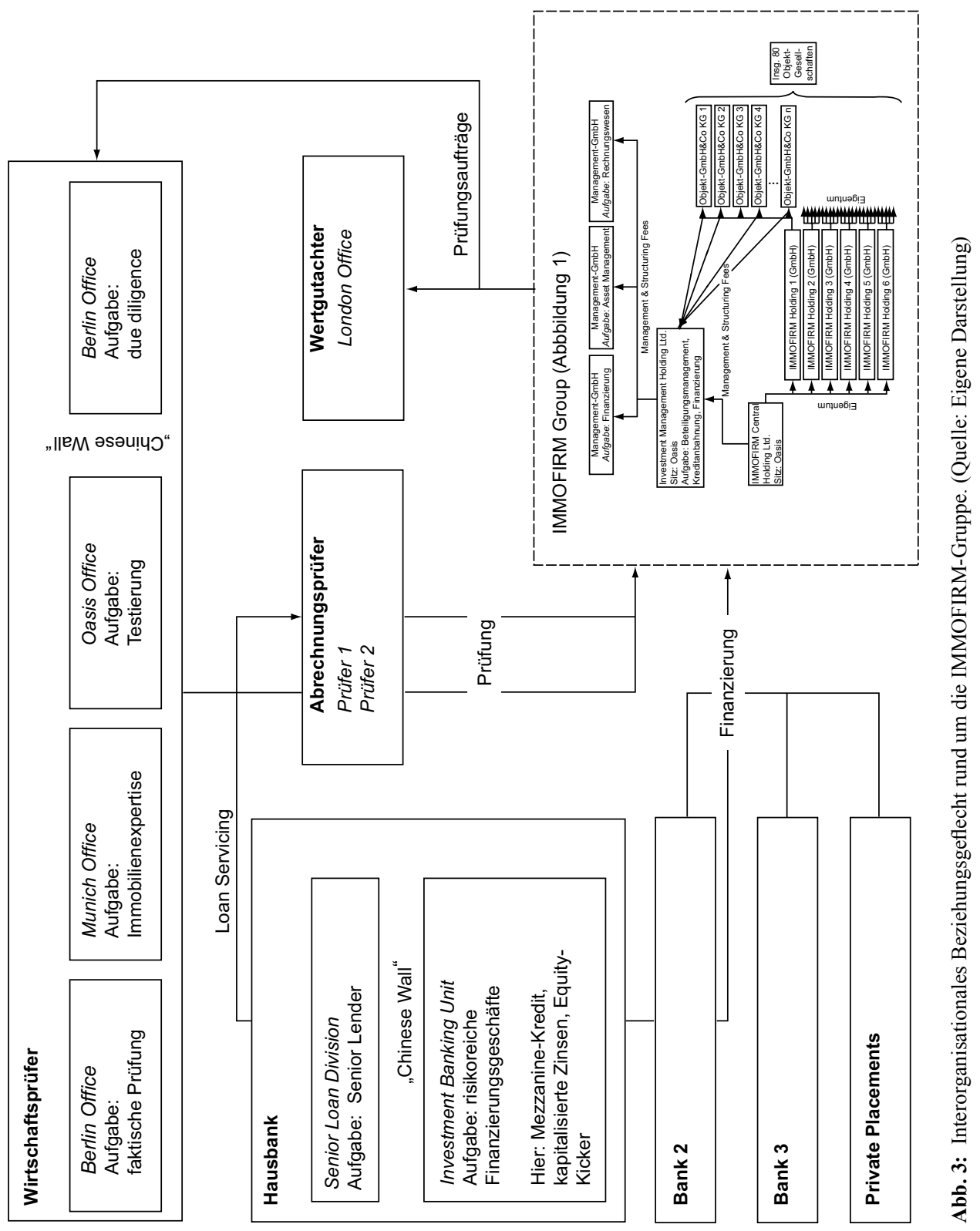


auch testieren.... Und die haben dem [deutschen Prüfer] wörtlich gesagt, er kann das Gutachten entweder akzeptieren oder tatsächlich das Testat verweigern. Und auf das, dass er das Testat verweigert, hat er sich nicht eingelassen, weil die [Wertgutachter Firma ABCD] ist auch nicht irgendwer am Markt.

Zusammenfassend lassen sich einige Charakteristika des Falls IMMOFIRM hervorheben: Die Struktur des Unternehmens ist äußerst fragmentiert und der formale, juristische Sitz bestimmter Unternehmensteile richtet sich vor allem nach steuerrechtlichen Erwägungen. Der Geschäftsprozess ist in viele verschiedene Module aufgegliedert, bei denen der Erwerb und das Betreiben von Immobilien nur von untergeordneter Bedeutung sind. Die finanzialisierte Wertschöpfungskette wird von unterschiedlichen, ebenfalls fragmentierten Dienstleistungsunternehmen zusammengefügt; ohne diese ließe sich das Geschäftsmodell von IMMOFIRM nicht realisieren. Im Kern geht es um die Kreation von Kapital mit Hilfe von Immobilien. Dieses Ziel ist ohne ein engmaschig gewobenes Netzwerk von Dienstleistungsanbietern nicht umzusetzen und basiert zu einem guten Teil auf kontinuierlich steigenden Bewertungen der einmal erworbenen Immobilien sowie deren Realisierung in Form von Refinanzierungstransaktionen.

\section{Diskussion des IMMOFIRM Falls}

In der Finanzwissenschaft dominierte lange die Annahme, Verbriefung und Weiterverkauf von Forderungen, wie zum Beispiel Asset-backed-Securities, führten dazu, Unsicherheiten in kalkulierbare Risiken zu transformieren. Auf dieser Welle wollte auch IMMOFIRM surfen, als die Manager beschlossen, Immobilien kreditfinanziert zu kaufen, sie in Unternehmen zu überführen und zum Abschluss das Unternehmen an die Börse zu bringen. Damit verkörperte auch dieses spezifische Geschäftsmodell den innovativen Charakter vieler Finanzinstrumente, die Risiken vermeintlich transparent und handelbar zu machen.

Der Fall IMMOFIRM zeigt damit auch, dass die Finanzindustrie vor dem abstrakten Hintergrund finanzökonomischer Annahmen ein erhebliches Eigenleben führen konnte. Statt einer Beherrschung ökonomischer Risiken kam es zu einem Over-leveraging, einer (wohl bewusst in Kauf genommenen) überoptimistischen Kalkulation der Risiken auf der einen und der erhofften Erträge von Banken und beteiligten Dienstleistern auf der anderen Seite. Gemeinschaftlich schielten alle Akteure auf die Blase, die es auszubeuten gelte („Der Markt ist bullish!“) und die durch kurze Zyklen der Kapitalbeschaffung (,Das Zauberwort war Refinanzierung") dadurch auch in ihrem Wachstum befeuert wurde. Die Basis für den zumindest kurzfristigen Erfolg dieser Geschäftspraktiken war das performative Zusammenwirken von gutachterlichen Bewertungsmodellen und bankseitigen Finanzierungspraktiken, die wechselseitig aufeinander bezogen sind: Kalkulatorische Wertsteigerungen von Immobilien erlaubten mit Hilfe von Refinanzierungskrediten den Kauf weiterer Immobilien, was wiederum zu weiteren kalkulatorischen Wertsteigerungen beitrug. Auf der Marktebene führte die Zunahme derartiger Geschäftspraktiken im Zeitraum zwischen 2004 und 2007 zu einer enormen Zunahme an (auch: Mehrfach-) Verkäufen von Wohnimmobilien: Wurden zwischen 1999 und 2003 nur zwischen 50000 und 150000 Wohnungen pro Jahr veräußert, waren es in dieser Hochphase zwischen 
280000 und 360000 Wohnungen jährlich (vgl. Claßen und Zander 2010, S. 379). In der Zeit vor der Finanzkrise war also nicht Kapital, sondern Zeit die knappste Ressource. Mit großer Hektik wurden Immobilien erworben und dafür mehr Kapital bereitgestellt, als den Immobilien lange Zeit als „Wert“ zugeschrieben worden war. Die einsetzende Krise beendete die Blase am Immobilienmarkt (vgl. Claßen und Zander 2010) und führte im Fall von IMMOFIRM zu einer Einstellung dieser Art der Geschäftstätigkeit. Eine konservativere Kreditvergabe durch die Banken hätte wohl schon zum Zeitpunkt des geplanten Immobilienerwerbs einen Großteil der Transaktionen nicht zustande kommen lassen.

Dass die bei IMMOFIRM beobachteten Geschäftspraktiken kein exotischer Einzelfall sondern, im Gegenteil, exemplarisch für neuere Entwicklungen gerade am deutschen Markt für Wohnimmobilien sind, zeigen die von Holm (2010) untersuchten Fälle Cerberus und Deutsche Annington. Insbesondere Private Equity Fonds wie Cerberus orientieren sich Holm (2010, S. 400) zu Folge ,weniger an traditionellen Bestandsstrategien als vielmehr an einer finanzmarktbezogenen Bilanzsteigerung der Anlagewerte.“ Als Mittel dienen dafür, wie auch im Fall von IMMOFIRM, „Neuordnungen von Kreditlinien und Neubewertungen der Wohnungsbestände“ (ebd.). Und für den Bereich von Geschäftsimmobilien dokumentiert Seidel (2010, S. 52 ff.) in seinem journalistischen Buch „Arcandors Absturz" ebenfalls, wie im Zusammenwirken mit Finanzdienstleistern, in diesem Fall die Kölner Bank Sal. Oppenheim, ein Mischkonzern wie KarstadtQuelle über den Umweg von Immobilienfonds finanzialisiert wurde. Die Immobilien von KarstadtQuelle wurden an eine Vielzahl eigens gegründeter Immobilienfonds verkauft und von diesen zurückgemietet, wobei im Zuge dieses Fragmentierungsprozesses wiederum hohe Profite auf Grund von Gebühren für die beteiligten Dienstleister anfielen.

Der Fokus auf Interorganisationsbeziehungen illustriert die Bedeutung von Dienstleistungsunternehmen für diese Finanzialisierung der Immobilienwirtschaft in dreifacher Weise. Zum ersten ermöglichen Dienstleistungsunternehmen die Trennung von Rechenschaft und Profit, indem sie die Organisation fragmentieren und neu zusammensetzen. Zum zweiten kreieren sie durch die Ermittlung und Testierung von Wertgutachten kalkulatorische Artefakte. Zum dritten schöpfen sie aus fiktivem Wert reales Kapital, indem sie Gebühren erheben, oder zu zahlen bereit sind, und diese in den Wirtschaftskreislauf einspeisen.

Die Analyse des Falls verweist, gerade auch in der Zusammenschau mit den von Holm (2010) und Seidel (2010) dokumentierten Vergleichsfällen, vor allem auf zwei Befunde hinsichtlich der Konfiguration interorganisationaler Beziehungen. Als erstes Ergebnis ist die Bedeutung der Gebühren (Fees) hervorzuheben. Sie sind auf zwei Ebenen relevant: Zum einen dienen sie IMMOFIRM zur Steuerminderung. Sie sind gewissermaßen Verrechnungspreise im internen Gefüge der Unternehmung und erlauben das „Verflüssigen“ eigentlich in Immobilien gebundenen Kapitals. Zum anderen werden Gebühren an externe Dienstleister gezahlt; sie werden zum ,Schmiermittel“ der Finanzialisierung des europäischen Immobiliensektors. Fees werden früh im Prozess der Kreation der Unternehmung fällig, und zwar wieder auf zweifache Weise. Einerseits fallen sie gegenüber Externen für Notar-, Beratungs-, Bewertungs-, Kreditierungs- und Prüfungsleistungen an. Anderseits dienen sie dem Unternehmensmanagement, das Buchhaltungs- und Finanzierungsleistungen innerhalb des Konglomerats mit Hilfe internationaler Rechtsanwaltskanzleien derart organisiert, dass Erträge als Fees definiert werden, die ausschließlich in Steueroasen anfal- 
len. Es handelt sich um die vorgezogene „Ausschüttung“ noch nicht realisierter Gewinne, also um die Kreation von Kapital mit Hilfe von branchenüblichen kalkulativen Praktiken.

Eine Ermittlung des Gesamtbetrags aller geleisteten Fees ist kaum möglich. Bedenkt man allerdings die Vielfältigkeit der Tätigkeiten und ihre Häufigkeit und berücksichtigt den rechnerischen Wert des Immobilienportfolios im Milliarden-Euro-Bereich, legt dies nahe, dass es sich um Gebühren im hohen, bis zu zweistelligen Millionenbereich gehandelt haben könnte - wohlgemerkt immer, ohne dass sich am eigentlichen Investitionsobjekt, den Immobilien, Maßgebliches geändert hätte.

Zum zweiten fällt ins Auge, dass die Verfügbarkeit von Kapital, zumindest in Zeiten des Booms, den Geschäftstätigkeiten der IMMOFIRM keine Grenzen setzte. Eher umgekehrt kann die Kreditvergabe der Banken als lax und unkritisch bezeichnet werden. Ziel der Kreditvergabe waren weniger langfristige Investments in Immobilien als vielmehr die Generierung eines Produkts, eben von Asset-Backed-Securities. Im Vordergrund stand der Aufbau des Immobilienportfolios und nicht seine (langfristige) Qualität, die in Zeiten des Booms auch nicht hinterfragt wurde. Ein derartiges Muster ist deshalb auch keineswegs auf den Immobilienmarkt beschränkt, sondern überall dort möglich, wo es zu assetbasierter Blasenbildung kommen kann, also beispielsweise auch in Rohstoffmärkten oder im Nahrungsmittelbereich.

Die Banken kooperierten in diesem Zusammenhang mit externen Gutachtern, die „objektivierte“ Preise bereitstellten. Der Wettbewerb von Gutachtern untereinander dürfte auch dazu beitragen, dass die Ergebnisse den Erwartungen der Auftraggeber nahe kommen. Die Bereitstellung von Kapital ist in hohem Maße durch eine Verschiebung und damit Externalisierung von Verantwortung gekennzeichnet: Banken vertrauen Gutachtern, kleinere Banken orientieren sich an größeren Banken. Kapitalerhöhungen werden mit Wertsteigerungen gerechtfertigt. An keiner Stelle schienen die Plausibilität des Geschäftsmodells oder die entstehenden systemischen Risiken überprüft oder reguliert $\mathrm{zu}$ werden. Das Augenmerk der handelnden Akteure des Immobiliensektors lag ausschließlich auf einem kurzfristigen, in manchen Fällen an ein Pyramidenspiel erinnernden Finanzierungsgebaren.

In diesem Zusammenhang mutet die Betonung der „Chinese Wall“" durch die beteiligten Akteure merkwürdig an: Unternehmen, die mit Grenzüberwindung ihre Profite generieren, schaffen innerhalb ihrer Organisation Grenzen, von denen sie behaupten, dass sie undurchlässig seien. In diesem Bereich sehen wir noch großen Bedarf für weitergehende Forschung in zweifacher Hinsicht. Einerseits gilt es genauer zu untersuchen, wie derartige „Chinese Walls“ in alltäglichen Geschäftspraktiken realisiert und aufrecht erhalten werden. Andererseits wären die Folgen eines durch derartige Konstruktionen erhöhten Risikos von zwei unabhängig agierenden Abteilungen innerhalb eines organisationalen Zusammenhangs zu untersuchen.

\section{Fazit und Ausblick: Finanzialisierung aus interorganisationaler Sicht}

Die Darstellung des Aufbaus eines europaweit operierenden Immobilienkonglomerats verdeutlicht zunächst vor allem die hohe Komplexität von Finanzmarktgeschäften. Genauer könnte man sagen, sie zeigt die notwendige Komplexität, um Wert kalkulieren 
und schaffen zu können. Da „finanzialisierte Wertschöpfung“ vor allem durch das Ausschöpfen steuerlicher und juristischer Grauzonen erfolgt, ist die Aktivität global operierender Dienstleistungsfirmen unerlässlich. Sie bringen ihre spezifischen Kenntnisse ein, die ursprünglich, wie bei Kanzleien oder Wirtschaftsprüfern, aus Arbitragegeschäften erwachsen sind. Unter der Ägide von Unternehmern, die eine große Bank zur Vergabe des „Senior Loans“ bewegen müssen, werden die unterschiedlichen Dienstleistungskompetenzen auf eine Art und Weise kombiniert, die die in erster Linie kalkulatorische Zuwächse unabhängig von realen Investitionen (wie z. B. Sanierungen) hervorbringt. Die projektbezogene Kooperation unterschiedlicher Unternehmen der Finanzdienstleistungsbranche (Banken, Makler, Notare, Wirtschaftsprüfer, Kunden) ist durch eine doppelte Verschiebung gekennzeichnet: Zum einen organisieren Dienstleistungsunternehmen die Verschiebung von Steuerpflicht und Haftung in diejenigen Jurisdiktionen, die aus Sicht der Beteiligten die bestmöglichen Bedingungen vorsehen. Diese Dynamiken sind politisch bedenklich, aber lange bekannt (Race-to-the-bottom, Delaware-Effekt). Zum anderen werden Profite sowohl in zeitlicher als auch in organisatorischer Hinsicht verschoben.

Hinsichtlich der ersten Verschiebung zeigt die vorliegende Studie, dass den Dienstleistungsunternehmen des Finanzsektors eine weitaus größere Bedeutung zukommt, als vielfach angenommen wird. Spezialisierte Dienstleister agieren deshalb nicht als bloße Vermittler, die Investoreninteressen folgen, bündeln oder umsetzen. Finanzdienstleistungsunternehmen sind vielmehr konstitutives Element finanzialisierten Wirtschaftens und lassen durch ihr spezialisiertes Angebot erst einen Großteil der Nachfrage entstehen, die sie selbst zu einem späteren Zeitraum bedienen. Die spezialisierten Finanzdienstleister operieren in der Regel international und machen sich den Wissensvorsprung, den sie durch die Kenntnis unterschiedlicher, in verschiedenen Ländern existierender Praktiken haben, zu Nutze. Sie sind in der Lage, bestehende rechtliche Unterschiede zwischen Jurisdiktionen auszunutzen und Dienstleistungen anzubieten, die nicht zuerst den operativen Erfordernissen ihrer Kunden entspringen; stattdessen stellen sie auf die gezielte Ausbeutung steuerlicher und regulatorischer Differenzen ab (Arbitragegeschäfte).

Den Akteuren des untersuchten Immobilienkonglomerats gelingt es erst mit Hilfe von Dienstleistungsunternehmen den Kern ihres Geschäftsmodells, nämlich die Finanzialisierung bestehender Wohnimmobilien, umzusetzen. Dienstleister sind in zweifacher Hinsicht unabdingbar: Erstens ermöglichen sie den Aufbau des Konglomerats als einen über mehrere Länder verteilten Verbund von Einzelunternehmen, die ihre Geschäftstätigkeit an demjenigen Ort erbringen, an dem sie die günstigsten Konditionen vorfinden. Zweitens stellen ihre Dienstleistungen in Form von Gutachten, Bilanzen, Verträgen oder Testaten jene Bausteine bereit, die die Fragmentierung der Unternehmenstätigkeit überwinden. Die erfolgreiche Überbrückung nationaler und organisationaler Grenzen wird zu einem Kernelement für die Entstehung finanzialisierter Unternehmen. Dienstleistungsunternehmen managen und legitimieren diese Überbrückung in Form von interorganisationalen Beziehungen und verkörpern daher mehr als bloß die „globale Infrastruktur“ des Finanzmarktkapitalismus (Beyer 2009, S. 313) - auch wenn sie diese Funktion weiterhin erbringen. Stattdessen sind sie aktiv an der Entstehung neuer, auf Finanzialisierung ausgerichteter Geschäftsmodelle beteiligt.

Zusammengefasst lässt sich feststellen, dass Dienstleister im untersuchten Fall eine „finanzialisierte Wertschöpfung“ ermöglichen, sie sogar von Grund auf organisieren. Das 
Beispiel ist damit jenseits der Transformation von Produktionsstrukturen angesiedelt und zeigt die zentrale Rolle von Finanzdienstleistungsfirmen. Sie sind elementar für die Entstehung von Unternehmen und die Organisation ihres Geschäftsmodells, deren Hauptzweck nicht in der Bereitstellung neuer Güterproduktion liegt, sondern die Erschließung von Anlagekapital ist. Solche Dienstleister schaffen nicht nur zusätzliches Angebot für nationale und internationale Anleger. Sie tragen zugleich zur Finanzialisierung von Bereichen des öffentlichen Lebens bei, die, wie bestehende mittelgroße Wohnimmobilien in Deutschland, zuvor nicht eng an die Finanzmärkte gekoppelt waren.

Konzeptionell bedeutsamer noch ist aber die zweite, temporale Verschiebungsdynamik, die anhand des Fallbeispiels gezeigt werden kann: Finanzialisierung zeigt sich hier als Vorziehung der Profitausschüttung. Dabei spielt die Kalkulation (Ermittlung, Berechnung und Legitimierung) von Gebühren eine zentrale Rolle. Letztere verkörpern eine neue, akkumulationsbasierte Rationalität (vgl. Krippner 2005) mit performativen, Blasenbildung begünstigenden Effekten. Sie wirken als zeitlich vorgezogene Ausschüttungen noch nicht realisierter Gewinne. Anders als Zinsen, die über die Kreditlaufzeit verteilt anfallen und aus laufenden Erträgen getilgt werden müssen, werden Gebühren auf einen fiktiven, aber von den Beteiligten als legitim anerkannten Wert bezogen und dann ausgeschüttet, wenn die (Beratungs-)Dienstleistungen anfallen. Auf diese Weise über kalkulatorische Profite legitimierte Werte gehen wiederum in Kreditvergabepraktiken ein und ermöglichen weitere Transaktionen, die wiederum kalkulatorische Profite zur Folge haben.

Diese spezifische Bedeutung von Gebühren als vorgezogene Ausschüttung kalkulierter Gewinne stellt aus unserer Sicht eine zentrale Erweiterung der eingangs erörterten Finanzialisierungsliteratur dar, da Dienstleistungsgebühren mehrere Funktionen erfüllen: Sie dienen zugleich der Steuervermeidung, der Vorziehung von Erträgen sowie der „Produktion“ von Kapital. Selbst ohne den (letztlich nicht zustande gekommenen) Börsengang sind im hier untersuchten Fall bereits hohe Beträge von Dienstleistungsintermediären verdient und entsprechende Bonuszahlungen an zuständige Akteure ausbezahlt worden. Selbstverständlich sind Gebühren, trotz der fiktiven Werte auf denen sie basieren, sehr real. Damit wird kalkuliertes Kapital in reales Kapital umgewandelt, das bei den Dienstleistern Gewinne und Boni erhöht. Damit erhöht sich die Bedeutung des Finanzsektors insgesamt und es wird Blasenbildung befördert, da Kapital unabhängig von tatsächlichen späteren Rückflüssen aus den getätigten Immobilieninvestitionen bereit gestellt wird. Fees untermauern die volkswirtschaftliche Relevanz des Finanzsektors im Allgemeinen und versetzen die Unternehmen in die Lage, hohe Gehälter zu zahlen und Gewinne auszuschütten. Aus fiktivem Kapital wird reales Kapital.

Kalkulatorische Praktiken stellen ein wichtiges Element der Finanzialisierungsliteratur dar, wenngleich bisher vor allem die Transformation des Produktionssektors im Mittelpunkt stand (vgl. Froud et al. 2006; Kädtler 2009). Das Fallbeispiel, das sich gerade dadurch auszeichnet, dass keine „materiellen Werte“ geschaffen wurden, sondern bloß vorhandene Immobilien erworben, neu arrangiert und an die Finanzmärkte weitergereicht werden sollten, ist noch in anderer Hinsicht instruktiv. Die Kalkulation, wie sie sich in Wertgutachten, Steuererklärungen und Testaten verbirgt, begründet eine organisierte Sozialordnung auf der Basis einer Wirklichkeitsproduktion durch Zahlen. Entscheidend sind, neben den Artefakten selbst, jene Organisationen, aus denen sich die kalkulato- 
rischen Infrastrukturen (vgl. Mennicken et al. 2008) zusammensetzen. Die Legitimität international operierender Dienstleistungsintermediäre ist eine wichtige Ressource für die Kalkulation von Preisen. Erst die Kombination von sachverständiger Beratung und Begutachtung auf der einen und ihre Erbringung durch anerkannte Firmen auf der anderen Seite, erklärt Bestand und Akzeptanz der Zahlenwerke, auf deren Basis die interorganisationalen Beziehungen ruhen können. Finanzdienstleister schaffen somit Organisationen, deren einziges Ziel es ist, Objekte für Anlagekapital sein zu können. Sie tun dies unter anderem durch die Interpretation, Repräsentation und Validierung von Zahlenwerken.

Aus übergeordneter Perspektive globalisierter Kapitalmärkte stellt sich die Problematik einer zeitlichen Verschiebung, der Vorholung von Erträgen, in noch größerer Form, da gerade hier Systemeffekte wirksam werden. Das neue Emergenzniveau (Ortmann 2010) ist daher nicht nur der Kumulation von Einzelakten geschuldet, sondern wird von einem stetig wachsenden Sektor der Finanzdienstleistungen zusätzlich befeuert. Anders lassen sich die erstaunlichen Wachstumsraten des Finanzsektors nicht erklären, der lange in der Lage schien, sich dauerhaft von den Wachstumswerten produzierender Sektoren abzusetzen. Das Platzen der Blase zeigt aber auch, dass die vorgezogene Gewinnausschüttung durch Fees nicht immer weiter laufen kann. Im Gegenteil zeigen die staatlich finanzierten Bankrettungspakete, dass eine Sozialisierung der Verluste in Zeiten der Krise auf die vorgezogene Gewinnausschüttung in Zeiten des Booms folgt. Aber auch für die Krisenreaktion werden Finanzdienstleister benötigt, die mehr sind als eine Infrastruktur des Finanzmarktkapitalismus. Sie sind einer seiner entscheidenden Treiber.

Danksagung: Wir danken Natalia Besedovski, Robert Müller und Arndt Sorge sowie dem Herausgeberkreis und zwei anonymen Gutachtern für hilfreiche Kommentare zu diesem Manuskript.

\section{Anhang A: Zur Rechtsform der GmbH \& Co KG}

Bei der Rechtsform der GmbH \& Co KG handelt es sich um eine Kommanditgesellschaft (KG, Rechtsgrundlage sind $\S \S 161$ bis $\S \S 177$ a HGB). In der Systematik des Gesellschaftsrechts zählen KGs zur Gruppe der Personengesellschaften und bestehen immer aus mindestens einem unbeschränkt und persönlich haftenden Komplementär und einem oder mehreren Kommanditisten, die nur mit ihren Einlagen haften.

Im Falle der $\mathrm{GmbH} \& \mathrm{Co} \mathrm{KG}$ ist jedoch der Komplementär eine GmbH. Es handelt sich also effektiv um eine haftungsbeschränkte Kapitalgesellschaft im rechtlichen Gewand einer Personengesellschaft. Gründe für die Wahl einer GmbH \& Co KG sind häufig steuerrechtliche Vorzüge in Verbindung mit Haftungsbeschränkungsstrategien.

\section{Anhang B: Glossar}

Arbitragegeschäft: Ausnutzen von rechtlichen oder steuerlichen Unterschieden für vergleichbare Waren in verschiedenen Jurisdiktionen Asset-backed-Securities: Verzinstes, forderungsbesichertes Wertpapier, dessen Zahlungsansprüche durch einen Bestand an Forderungen (assets) gedeckt (backed) sind 
Asset-Management: Finanzanlageentscheidungen im Rahmen von Dienstleistungen zur Vermögensverwaltung

Cash-Pooling: Liquiditätsbündelung durch Kredite zwischen verschiedenen Gesellschaften innerhalb eines Konzernverbunds mit einheitlicher Leitung

Chinese Wall: Organisationale Trennung von Abteilungen innerhalb eines Unternehmens zur Vermeidung von Interessenskonflikten auf Grund unterschiedlicher Zielsetzungen Credit Default Swaps: Kreditderivat, das Ausfallsrisiken von Krediten handelbar macht Due Diligence: Rechtliche und wirtschaftliche Prüfung von Anlagegütern vor deren Kauf (engl. für ,gebotene Sorgfalt")

Eigenkapital/equity: Gesellschaftsvermögen nach Abzug sämtlicher Verbindlichkeiten Equity Kicker: Klausel in einem Kreditvertrag, der Zahlungen im Falle eines Verkaufs oder Börsengangs vorsieht

GmbH-Komplementär: siehe AnhangA

Holding-Gesellschaft: Bezeichnung einer Gesellschaft, die der Verwaltung mehrer Tochtergesellschaften dient, die im Eigentum der Holding-Gesellschaft stehen

Komplementärin: Unbeschränkt haftender Gesellschafter einer Kommanditgesellschaft, siehe auch Anhang A

Loan-to-Value: Kreditvergabepraxis, die sich am gutachterlich ermittelten Wert des finanzierten Objekts orientiert

Management \& Structuring Fees: Gebühren, die für Management- und Finanzierungsdienstleistungen an eine Muttergesellschaft oder eine Dienstleistungsgesellschaft gezahlt werden, häufig in Form umsatzbezogener Verrechnungspreise

Offshore-Gesellschaft: Gesellschaft mit Sitz in einem Gebiet mit niedrigeren Steuersätzen und Regulierungsstandards als im Staat der eigentlichen Geschäftstätigkeit wie z. B. Kanalinseln oder Liechtenstein

Over-leveraging: Eingehen zu hoher Verbindlichkeiten, die nicht mehr bedient werden können

Ponzi finance: Bezeichnung Hyman Minskys für Finanzierungspraktiken, bei denen die laufenden Einkünfte nicht einmal mehr ausreichen, um die Zinsen eines Kredits zu bedienen; in Anlehnung an die amerikanische Bezeichnung für Pyramidenspiele und Schneeballsysteme („Ponzi Scheme“)

Share Deals: Verkauf nicht der gesamten Immobilie sondern nur eines Anteils der Inhabergesellschaft, um Grunderwerbssteuern zu sparen

Steueroase: siehe Offshore-Gesellschaft

Sub-Prime-Markt: Umgangssprachliche Bezeichnung für Handel mit verbrieften Paketen von Hypothekarkrediten mit geringerer als der höchsten Bonitätsstufe

Zweckgesellschaften: Gesellschaften, die nur für einen einzigen, engen Zweck gegründet werden (z. B. zur Auslagerung bestimmter Vermögenspositionen)

\section{Literatur}

Ansell, Christopher K., und Steven Weber. 1999. Organizing international politics: Sovereignty and open systems. International Political Science Review 20:73-93. 
Beyer, Jürgen. 2009. Varietät verspielt? Zur Nivellierung der nationalen Differenzen des Kapitalismus durch globale Finanzmärkte. Kölner Zeitschrift für Soziologie und Sozialpsychologie 49:305-325.

Botzem, Sebastian. 2012. The politics of accounting regulation. Organizing transnational standard setting in financial reporting. Cheltenham: Edward Elgar.

Boyer, Robert. 2007. Assessing the impact of fair value upon financial crisis. Socio-Economic Review 5:779-807.

Claßen, Gudrun, und Christoph Zander. 2010. Handel mit Mietwohnungsportfolios in Deutschland: Umfang und Auswirkungen vor dem Hintergrund von Internationalisierung und Professionalisierung. Informationen zur Raumentwicklung 5:377-390.

Davis, Gerald F., Kristina A. Diekmann und Catherine H. Tinsley. 1994. The decline and fall of the conglomerate firm in the 1980s: The deinstitutionalization of an organizational. American Sociological Review 59:547-570.

Deeg, Richard. 2010. Institutional change in financial systems. In The Oxford handbook of comparative institutional analysis, Hrsg. Glenn Morgan, John Campbell, Colin Crouch, Peer Hull Kristensen, Ove Kai Pedersen und Richard. Whitley, 309-334. Oxford: Oxford University Press.

Deutschmann, Christoph. 2005. Finanzmarkt-Kapitalismus und Wachstumskrise. In FinanzmarktKapitalismus. Analysen zum Wandel von Produktionsregimen, Sonderheft 45 der Kölner Zeitschrift für Soziologie und Sozialpsychologie, Hrsg. Paul Windolf, 58-84. Wiesbaden: VS Verlag für Sozialwissenschaften.

Epstein, Gerald A. 2005. Introduction: Financialization and the world economy. In Financialization and the world economy, Hrsg. Gerald A. Epstein, 3-16. Cheltenham: Edward Elgar.

Erturk, Ismail, Julie Froud, Johal Sukhdev, Adam Leaver und Karel Williams. 2007. The democratization of finance? Promises, outcomes and conditions. Review of International Political Economy 14:553-575.

Froud, Julie, Johal Sukhdev, Adam Leaver und Karel Williams. 2006. Financialization and strategy: Narrative and numbers. London: Routledge.

Garud, Raghu, und Peter Karnøe. 2003. Bricolage versus breakthrough: Distributed and embedded agency in technology entrepreneurship. Research Policy 32:277-300.

Heires, Marcel, und Andreas Nölke. 2011. Finanzkrise und Finanzialisierung. In Die Internationale Politische Ökonomie der Weltfinanzkrise, Hrsg. Oliver Kessler, 37-52. Wiesbaden: VS Verlag für Sozialwissenschaften.

Helleiner, Eric. 2011. Understanding the 2007-2008 global financial crisis: Lessons for scholars of international political economy. Annual Review of Political Science 14:67-87.

Holm, Andrej. 2010. Institutionelle Anbieter auf deutschen Wohnungsmärkten -- neue Strategien der Wohnraumbewirtschaftung. Informationen zur Raumentwicklung 5:391-402.

Höpner, Martin. 2003. Wer beherrscht die Unternehmen? Shareholder value, Managerherrschaft und Mitbestimmung in Deutschland. Frankfurt a. M.: Campus.

Jacobides, Michael G. 2005. Industrie change through vertical dis-integration: How and why markets emerged in mortgage banking. Academy of Management Journal 48:465-498.

Jürgens, Ulrich, Joachim Rupp und Katrin Vitols (unter Mitarbeit von Bärbel Jäschke-Werthmann). 2000. Corporate governance and shareholder value in Deutschland. Discussion Paper FS II 00-202, 2000. Berlin: Wissenschaftszentrum Berlin für Sozialforschung.

Kädtler, Jürgen. 2009. Finanzialisierung und Finanzmarktrationalität. Zur Bedeutung konventioneller Handlungsorientierungen im gegenwärtigen Kapitalismus. SOFI Arbeitspapier 2009-5. Göttingen: Soziologisches Forschungsinstitut (SOFI).

Krippner, Greta R. 2005. The financialization of the American economy. Socio-Economic Review 3:173-208.

Krippner, Greta R. 2011. Capitalizing on crisis: The political origins of the rise of finance. Cambridge: Harvard University Press. 
Leipold, Klaus. 2008. Die Strafbarkeit von Unternehmen. Neue juristische Wochenschrift 68 (Spezial, Heft 7) :216-217.

MacKenzie, Donald, und Yuval Millo. 2003. An equation and its worlds: Bricolage, exemplars, disunity and performativity in financial economics. Social Studies of Science 33:831-868.

MacKenzie, Donald, Fabian Muniesa und Lucia Siu, Hrsg. 2007. Do economists make markets? On the performativity of economics. Princeton: Princeton University Press.

Mennicken, Andrea, und Hendrik Vollmer, Hrsg. 2007. Zahlenwerk. Kalkulation, Organisation und Gesellschaft. Wiesbaden: VS-Verlag für Sozialwissenschaften.

Mennicken, Andrea, Peter Miller und Rita Samiolo. 2008. Accounting for economic sociology. Economic Sociology. The European Electronic Newsletter 10:3-7.

Minsky, Hyman P. 1986/2008. Stabilizing an unstable economy. New York: McGraw-Hill.

Münch, Richard, und Tina Guenther. 2005. Der Markt als Organisation. Von der Hegemonie der Fachspezialisten zur Hegemonie des Finanzmanagements. In Finanzmarkt-Kapitalismus. Analysen zum Wandel von Produktionsregime, Sonderheft 45 der Kölner Zeitschrift für Soziologie und Sozialpsychologie, Hrsg. Paul Windolf, 394-417. Wiesbaden: VS Verlag für Sozialwissenschaften.

Nölke, Andreas. 2009. Finanzkrise, Finanzialisierung und Vergleichende Kapitalismusforschung. Zeitschrift für Internationale Beziehungen 16:123-139.

Nölke, Andreas, und James Perry 2008. The power of transnational private governance: Financialization and the IASB. Business and Politics 9: Artikel 4.

Ortmann, Günther. 2010. Organisation und Moral: Die dunkle Seite. Göttingen: Velbrück Wissenschaft.

Pettigrew, Andrew M. 1990. Longitudinal field research on change: Theory and practice. Organizations Science 1:267-292.

Power, Michael. 2004. Counting, control and calculation: Reflections on measuring and management. Human Relations 57:765-783.

Rappaport, Alfred. 1998. Creating shareholder value. A guide for managers and investors. Revised and updated edition. New York: The Free Press.

Seidel, Hagen. 2010. Arcandors Absturz. Wie man einen Milliardenkonzern ruiniert: Madeleine Schickedanz, Thomas Middelhoff, Sal. Oppenheim und Karstadt Quelle. Frankfurt a. M.: Campus.

Semmler, Willi, und Lucas Bernard. 2009. Boom-bust cycles: Leveraging, complex securities, and asset prices. New School Working Paper. http://www.newschool.edu/nssr/cem/papers/ wp/financial/SemmlerBernardNewversionSept2709_mrLucas_4.pdf. (Zugegriffen: 27. Apr. 2007).

Sorge, Arndt. 2011. Financial catastrophe and its implications for socioeconomics. Review essay. Socio-Economic Review 9:169-186.

Streeck, Wolfgang, und Daniel Mertens. 2010. Politik im Defizit: Austerität als fiskalpolitisches Regime. Der moderne Staat 3:7-29.

Vollmer, Hendrik. 2003. Bookkeeping, accounting, calculative practice: The sociological suspense of calculation. Critical Perspectives on Accounting 14:353-381.

Vollmer, Hendrik. 2004. Folgen und Funktionen organisierten Rechnens. Zeitschrift für Soziologie $33: 450-470$.

Vollmer, Hendrik, Andrea Mennicken und Alex Preda. 2009. Tracking the numbers: Across accounting and finance, organizations and markets. Accounting, Organizations and Society 34:619-637.

Vormbusch, Uwe. 2004. Accounting. Die Macht der Zahlen im gegenwärtigen Kapitalismus. Berliner Journal für Soziologie 1:33-50.

Windolf, Paul, Hrsg. 2005a. Finanzmarkt-Kapitalismus. Analysen zum Wandel von Produktionsregimen. Sonderheft 45 der Kölner Zeitschrift für Soziologie und Sozialpsychologie. Wiesbaden: VS Verlag für Sozialwissenschaften. 
Windolf, Paul. 2005b. Die neuen Eigentümer. In Finanzmarkt-Kapitalismus. Analysen zum Wandel von Produktionsregimen, Sonderheft 45 der Kölner Zeitschrift für Soziologie und Sozialpsychologie, Hrsg. Paul Windolf, 8-19. Wiesbaden: VS Verlag für Sozialwissenschaften.

Windolf, Paul. 2005c. Was ist Finanzmarkt-Kapitalismus? In Finanzmarkt-Kapitalismus. Analysen zum Wandel von Produktionsregimen, Sonderheft 45 der Kölner Zeitschrift für Soziologie und Sozialpsychologie, Hrsg. Paul Windolf, 20-57. Wiesbaden: VS Verlag für Sozialwissenschaften.

Windolf, Paul. 2008. Eigentümer ohne Risiko. Die Dienstklasse des Finanzmarkt-Kapitalismus. Zeitschrift für Soziologie 37:516-535.

Windolf, Paul. 2009. Zehn Thesen zur Finanzmarkt-Krise. Leviathan 37:187-196.

Young, Brigitte. 2011. Der privatisierte Keynesianismus, die Finanzialisierung des ,alltäglichen Lebens' und die Schuldenfalle. In Die Internationale Politische Ökonomie der Weltfinanzkrise, Hrsg. Oliver Kessler, 15-36. Wiesbaden: VS Verlag für Sozialwissenschaften.

Sebastian Botzem, 1972, Dr. rer. pol., wissenschaftlicher Mitarbeiter, Projektgruppe „Modes of Economic Governance“ Wissenschaftszentrum Berlin für Sozialforschung. Forschungsgebiete: Internationale Politische Ökonomie, Transnationale Governance, Organisationsforschung und Regulierung der Finanzmärkte. Veröffentlichungen: The politics of accounting regulation. Organizing transnational standard setting in financial reporting. Cheltenham 2012; Standardization cycles. A process perspective on the formation and diffusion of transnational standards. Organization Studies 33, 2012 (mit L. Dobusch).

Leonhard Dobusch, 1980, Dr. rer. pol., Juniorprofessor für Organisationstheorie am Institut für Management des Fachbereichs Wirtschaftswissenschaft der Freien Universität Berlin. Forschungsschwerpunkte: Management digitaler Gemeinschaften, private Regulierung via Standardisierung, vor allem im Bereich des Immaterialgüterrechts, heterodoxe Ökonomie. Veröffentlichungen: Windows versus Linux. Wiesbaden, 2008; Interorganisationale Netzwerke und digitale Gemeinschaften: Von Beiträgen zu Beteiligung? Managementforschung 21, 2011 (mit S. Quack); Standardization cycles: A process perspective on the formation and diffusion of transnational standards. Organization Studies 33, 2012 (mit S. Botzem). 